\title{
A necessary condition for best fitting analytical to simulated density profiles in dark matter haloes
}

\author{
R. Caimmi*
}

December 8, 2017

\begin{abstract}
Analytical and geometrical properties of generalized power-law (GPL) density profiles are reviewed, and special effort is devoted to the special cases where GPL density profiles reduce to (i) a double power-law (DPL), and (ii) a single power-law (SPL). Then GPL density profiles are compared with simulated dark haloes (SDH) density profiles, and nonlinear least-squares fits are prescribed, involving five parameters (a scaling radius, $r_{0}$, a scaling density, $\rho_{0}$, and three exponents, $\alpha, \beta, \gamma$ ), which specify the fitting profile (RFSM5 method). More specifically, the validity of a necessary condition for the occurrence of an extremal point, is related to the existence of an intersection between three surfaces in a 3-dimension space. Using the algorithm makes also establish that the extremal point is a fiducial minimum, while the explicit calculation of the Hessian determinant is avoided to gain in simplicity. In absence of a rigorous proof, the fiducial minimum can be considered as nothing but a fiducial absolute minimum. An
\end{abstract}

*Astronomy Department, Padua Univ., Vicolo Osservatorio 2, I-35122 Padova, Italy email: caimmi@pd.astro.it 
application is made to a sample of $17 \mathrm{SDHs}$ on the scale of cluster of galaxies, within a flat $\Lambda$ CDM cosmological model (Rasia et al. 2004). In dealing with the averaged SDH density profile (ADP), a virial radius, $r_{v i r}$, equal to the mean over the whole sample, is assigned, which allows the calculation of the remaining parameters. The following results are found. (i) A necessary condition for the occurrence of an extremal point is satisfied for 8 sample haloes, and is not for the remaining 9 together with ADP. In the former alternative, an extremal minimum point (EMP) may safely exist. In the latter alternative, the occurrence of an EMP cannot be excluded, but only a non extremal minimum can be determined. (ii) The occurrence of an EMP implies a sum of square residuals which is systematically lower than its counterpart deduced by use of numerical RFSM5 methods. Accordingly, EMPs may safely be thought of as absolute minima. With regard to sample haloes where no EMP is detected, the above result maintains in 4 cases (including ADP), while the contrary holds for the remaining 5 cases. (iii) The best fit (with no EMP detected) is provided by DPL density profiles for 3 sample haloes. In addition, DPL density profiles make a rough, but viable approximation in fitting SDH density profiles. The contrary holds for SPL density profiles. No evident correlation is found between SDH dynamical state (relaxed or merging) and asymptotic inner slope of the logarithmic density profile or (for SDH comparable virial masses) scaled radius. Mean values and standard deviations of some parameters are calculated and, in particular, the decimal logarithm of the scaled radius, $\xi_{\text {vir }}$, reads $<\log \xi_{\text {vir }}>\approx 0.59$ and $\sigma_{s \log \xi_{v i r}} \approx 0.59$, the standard deviation exceeding by a factor 3.3-3.4 its counterpart evaluated in an earlier attempt using NFW (Navarro et al. 1995, 1996, 1997) density profiles (Bullock et al. 2001). If a large dispersion still maintains for richer samples, in dealing with analytical RSFM5 methods, a low dispersion found in $N$-body simulations seems to be an artefact, due to the assumption of NFW (or any equivalent choice) density profile. It provides additional support to the idea, that NFW density profiles may be considered as a convenient way to parametrize SDH density profiles, without implying that it necessarily produces the best possible fit (Bullock et al. 2001). With regard to RFSM5 methods formulated in the current paper, the exponents of both the best fitting GPL density profile to $\mathrm{ADP},(\alpha, \beta, \gamma) \approx(0.3,4.5,1.5)$, and related averages calculated over the whole halo sample, $(\bar{\alpha}, \bar{\beta}, \bar{\gamma}) \approx(3.0,3.9,1.3)$, are 
far from their NFW counterparts, $(\alpha, \beta, \gamma)=(1,3,1)$. The last result, together with the large value of the standard deviation, $\sigma_{s} \log \xi_{v i r}$, is interpreted as due to a certain degree of degeneracy in fitting GPL to SDH density profiles. If it is a real feature of the problem, or it could be reduced by the next generation of high-resolution simulations, still remains an open question. Values of asymptotic inner slope of fitting logarithmic density profiles, are consistent with results from recent high-resolution simulations (Diemand et al. 2004; Reed et al. 2004). keywords - cosmology: dark matter - galaxies: clusters:general.

\section{Introduction}

Recent observations from anisotropies in the cosmic microwave background, large-scale structure surveys, Hubble parameter determinations, and Type Ia supernovae results, allow narrow ranges for the values of cosmological quantities (e.g., Sievers et al. 2003; Rubiño-Martin et al. 2003; Spergel et al. 2003). The related $(\Lambda \mathrm{CDM})$ cosmological model is consistent with a bottom-up picture (hierarchical clustering) of dark matter haloes, where smaller systems formed first from initial density perturbations and then merged with each other to become larger systems, or were tidally disrupted and accreted from previously formed larger systems.

A two-parameter functional form for the halo profile, where a scaled density depends on a scaled radius and three exponents are specified, has been proposed by several authors (e.g., Navarro et al. 1995, 1996, 1997, hereafter quoted together as NFW; Moore et al. 1998, 1999; Rasia et al. 2004). Generally speaking, it may be considered as a special case of the 5-parameter family (Hernquist 1990):

$$
\rho\left(\frac{r}{r_{0}}\right)=\frac{\rho_{0}}{\left(r / r_{0}\right)^{\gamma}\left[1+\left(r / r_{0}\right)^{\alpha}\right] \chi} \quad ; \quad \chi=\frac{\beta-\gamma}{\alpha} ;
$$

where $\rho_{0}$ and $r_{0}$ are a scaling density and a scaling radius, respectively, and all the exponents are positive in the case under discussion.

The density profile, expressed by Eq. (1), reduces to a power-law both towards the centre, $r \rightarrow 0$, and towards infinite, $r \rightarrow+\infty$, where the exponent equals $-\gamma$ and $-\beta$, respectively. It may be conceived as a generalized power-law and, in the following, it shall be quoted as GPL density profile. 
On the other hand, matter distribution within a simulated dark matter halo, in the following, shall be quoted as SDH density profile.

For fixed exponents, one among the two remaining free parameters, the scaling density and the scaling radius, may be related to the mass and the radius of the virialized region. For further details see e.g., NFW; Bullock et al. (2001); Rasia et al. (2004); Caimmi et al. (2004; hereafter quoted as CMV).

Strictly speaking, GPL density profiles cannot be considered as universal, in the sense that a fixed choice of exponents, $(\alpha, \beta, \gamma)$, does not provide the best fit to an assigned set of SDH density profiles (e.g., Bullock et al. 2001; CMV). A weaker conception of universal, GPL density profiles, might be related to the insensitivity of the shape to halo masses, initial density fluctuation spectra, or cosmological parameters (NFW; Hess et al. 1999; Klypin et al. 2001; Bullock et al. 2001; Fukushige \& Makino 2001, 2003; Zhao et al. 2003).

However, no general consensus still exists on this point. In particular, some authors found an inner slope which depends on the power spectrum of the initial density perturbation (Syer \& White 1998), or on the mass (Jing \& Suto 2000; Hiotelis 2003; Ricotti 2003). Further support to the above results is provided by recent, high-resolution, $N$-body simulations (Fukushige et al. 2004; Navarro et al. 2004). In addition, a certain degree of degeneracy occurs, with regard to the exponents, $(\alpha, \beta, \gamma)$, in fitting various GPL to $\mathrm{SDH}$ density profiles, in the whole range of resolved scales (e.g., Klypin et al. 2001). For a more detailed discussion, see CMV.

According to the above remarks, GPL density profiles may be considered as a convenient way to parametrize SDH density profiles, without implying that it necessarily produces the best possible fit (Bullock et al. 2001). The search of the best fitting GPL to (one or more) SDH density profiles, makes the aim of the current paper. To this respect, different fitting criteria exist, such as minimizing the maximum fractional deviations of the fit, $\max \left|\log \left(\rho_{G P L} / \rho_{h}\right)-\log \left(\rho_{S D H} / \rho_{h}\right)\right|$ (e.g., Klypin et al. 2001); the sum of the squares of absolute ${ }^{1} \operatorname{logarithmic}$ residuals, $\chi^{2}=\sum\left[\log \left(\rho_{G P L} / \rho_{h}\right)-\right.$ $\left.\log \left(\rho_{S D H} / \rho_{h}\right)\right]^{2}$ (e.g., Bullock et al. 2001); the sum of squares of relative

\footnotetext{
${ }^{1}$ The term "absolute" here has not to be intended as "absolute value", but as opposite to "relative". More precisely, $y_{i}-y\left(x_{i}\right)$ is an absolute residual, while $\left[y_{i}-y\left(x_{i}\right)\right] / y\left(x_{i}\right)$ is the corresponding relative residual.
} 
residuals, $\sum\left[\left(\rho_{S D H} / \rho_{h}-\rho_{G P L} / \rho_{h}\right) /\left(\rho_{G P L} / \rho_{h}\right)\right]^{2}$ (e.g., Fukushige \& Makino 2003, 2004), where $\rho_{h}$ is a normalization value. For a more detailed discussion see e.g., Tasitsiomi et al. (2004).

The hierarchical collapse of dark matter into virialized haloes is likely to have played a key role in the formation of large-scale objects, such as galaxies and clusters of galaxies. The halo density distribution has a direct dynamical role in determining the observable parameters of the baryonic subsystems. Then further investigation even on the best fitting GPL to SDH density profile, appears to be important.

To this aim, a nonlinear least-squares method is used in the current paper. The related, general procedure, shall be quoted in the following as RFSM5 (Residuals Functions Sum Minimization within a 5-dimension hyperspace) method. The radius of the virialized region is taken from computer outputs, for selected cosmological parameters, which allows to deduce the mass of the virialized region, and the remaining quantities of interest. Further details may be found in an earlier attempt (CMV), where fiducial minima of the sum of absolute values and squares of residuals are calculated for assigned SDH density profiles, using a 5-dimension hypergrid within a 5-dimension hyperspace.

An analytical procedure, instead of the above mentioned numerical, shall be exploited in the current paper. The problem may be formulated from both an analytical and geometrical point of view. The sum of square residuals is a function of 5 variables in a 6-dimension hyperspace, $w=F\left(r_{0}, \rho_{0}, \alpha, \beta, \gamma\right)$ which, in turn, represents a 5-dimension hypersurface. The extremal point of absolute minimum defines the best fitting GPL to SDH density profile under consideration. If more than one extremal points of absolute minimum exist, then degeneracy occurs. For further details, see CMV.

According to a theorem of mathematical analysis, a necessary condition for the occurrence of an extremal point of minimum, is a null (or undefined) value of all the first partial derivatives of an assigned function, evaluated at that point. A study of the Hessian determinant enables to establish additional features (minimum, maximum, hyperflexion, hypersaddle). Finally, one has to ensure that the minimum is an absolute minimum. For further details on the analytic procedure see e.g., Smirnov (1969, Chap. V, $\S \S 2.2-2.6$ ).

In fitting GPL to SDH density profiles, our attention shall be restricted to the sum of square residuals, as absolute values exhibit a discontinuity of the first derivative for null arguments, which makes a solution of the problem 
much more difficult. An algorithm will be formulated and used, for searching points where the above mentioned necessary condition for the occurrence of an extremal minimum, is satisfied. Then it will be inferred, by comparison with numerical results found in an earlier attempt (CMV), that the related point is a fiducial minimum. On the other hand, no proof will be produced, that the point under discussion is an absolute minimum. This, and other improvements, are left to mathematicians.

The fit of GPL to SDH density profiles, which has deeply been investigated in CMV, is reviewed in section 2. The analytical RFSM5 method used for fulfilling the necessary condition for the occurrence of an extremal point of minimum, is presented in section 3 and derived in Appendix A. The subject of section 4 is an application to a sample of $17 \mathrm{SDH}$ and the related mean density profile, on the scale of clusters of galaxies, taken from Rasia et al. (2004), and already used in CMV. Then the results are discussed. Some concluding remarks are drawn in section 5 .

\section{Fitting GPL to SDH density profiles}

A detailed investigation on GPL and SDH density profiles, together with their comparison, has been performed in an earlier attempt (CMV), to which an interested reader is addressed. Only what is relevant for the current paper, or has not been mentioned in CMV, shall be reported here.

\subsection{GPL density profiles}

Plotting GPL density profiles on a logarithmic plane, $(\mathrm{O} \log \xi \log f$ ), necessarily implies use of dimensionless coordinates, defined as:

$$
\xi=\frac{r}{r_{0}} ; \quad f(\xi)=\frac{\rho}{\rho_{0}} ;
$$

where $\xi$ and $f$ can be conceived as a scaled radius and a scaled density, respectively. Accordingly, Eq. (1) reduces to:

$$
f(\xi)=\frac{1}{\xi^{\gamma}\left(1+\xi^{\alpha}\right)^{\chi}} \quad ; \quad \chi=\frac{\beta-\gamma}{\alpha} ;
$$

independent of the scaling parameters. 
As Eqs. (2) and (3) imply null density at infinite radius, the mass distribution has necessarily to be ended at an assigned isopycnic surface, which defines a truncation radius, $R$. The mass within the truncation isopycnic surface is (Caimmi \& Marmo 2003; CMV):

$$
M=M(R)=\frac{4 \pi}{3} r_{0}^{3} \rho_{0} \nu_{\text {mas }}=M_{0} \nu_{\text {mas }} ;
$$

where $M_{0}$ is a scaling mass and the profile factor, $\nu_{\text {mas }}$, has the explicit expression:

$$
\nu_{\text {mas }}=3 \int_{0}^{\Xi} f(\xi) \xi^{2} \mathrm{~d} \xi ;
$$

and the integration is carried up to:

$$
\Xi=\frac{R}{r_{0}}
$$

which may be conceived as a scaled, truncation radius.

Two limiting cases of Eq. (3) can be relevant to the aim of the current paper. The first one occurs as $\alpha \rightarrow+\infty$, which implies $\chi \rightarrow 0$ provided $\beta$ and $\gamma$ remain finite, and:

$$
\begin{aligned}
& \left(1+\xi^{\alpha}\right)^{\chi} \sim 1 ; \quad \xi \leq 1 ; \\
& \left(1+\xi^{\alpha}\right)^{\chi} \sim \xi^{\alpha \chi} \sim \xi^{\beta-\gamma} ; \quad \xi>1 ;
\end{aligned}
$$

accordingly, Eq. (3) reduces to:

$$
\lim _{\alpha \rightarrow+\infty} f(\xi)=\left\{\begin{array}{lll}
\xi^{-\gamma} ; & \xi \leq 1 ; & r \leq r_{0} ; \\
\xi^{-\beta} ; & \xi>1 ; & r>r_{0}
\end{array} ;\right.
$$

which is a double power-law with exponents equal to $-\gamma$ and $-\beta$.

The second limiting case of Eq. (3) occurs as $\beta \rightarrow+\infty$, which implies $\chi \rightarrow+\infty$ provided $\alpha$ and $\gamma$ remain finite. If, in addition, $r_{0} \rightarrow+\infty$ i.e. $\Xi \rightarrow 0$, and:

$$
\left(1+\xi^{\alpha}\right)^{\chi} \sim \frac{1}{C_{\xi}} ; \quad \rho_{0} \sim C_{\rho} \Xi^{\gamma} ;
$$

where $C_{\xi}$ and $C_{\rho}$ are constants, then Eq. (3) reduces to:

$$
\lim _{\beta \rightarrow+\infty} f(\xi)=\frac{C_{\xi}}{\xi^{\gamma}}
$$


A similar result is also obtained as $\alpha \rightarrow 0$ provided $\chi$ remains constant, which implies $\beta \rightarrow \gamma$ and:

$$
\left(1+\xi^{\alpha}\right)^{\chi} \sim 2^{\chi}
$$

accordingly, Eq. (3) reduces to:

$$
\lim _{\alpha \rightarrow 0} f(\xi)=2^{-\chi} \xi^{-\gamma}
$$

which is a power-law with exponent equal to $-\gamma$.

The logarithmic GPL density profile, deduced from Eq. (3), is:

$$
\log f=-\gamma \log \xi-\chi \log \left(1+\xi^{\alpha}\right)
$$

and the maximum variation in slope occurs at $\log \xi=\log \left(r / r_{0}\right)=0$ i.e. $r=r_{0}$, which discloses the geometrical meaning of the scaling radius in GPL density profiles. In the $\log$ arithmic plane (O $\log \xi \log f$ ), Eq. (10) represents a curve where two asymptotes exist:

$$
\begin{array}{ll}
\log f=-\gamma \log \xi ; & \xi \ll 1 ; \\
\log f=-\beta \log \xi ; & \xi \gg 1 ;
\end{array}
$$

which meet at the origin. For further details, see CMV.

\subsection{SDH density profiles}

Simulated haloes are characterized by a "virial" parameter, either the virial mass, $M_{v i r}$, or the virial radius, $r_{v i r}$, defined such that the mean density inside the virial radius is $\Delta_{v i r}$ times the mean matter universal density, $\rho_{h}=$ $\rho_{\text {crit }} \Omega_{m}$, at that redshift:

$$
M_{v i r}=\frac{4 \pi}{3} \Delta_{v i r} \rho_{c r i t} \Omega_{m} r_{v i r}^{3}
$$

where $\rho_{\text {crit }}=3 H^{2} /(8 \pi G)$ is the critical density for closure. The critical overdensity at virialization, $\Delta_{v i r}$, is motivated by the spherical collapse model: it is below two hundreds for an Einstein-de Sitter cosmology, and exceeds three hundreds for a flat $\Lambda$ CDM cosmology where $\Omega_{m} \approx 0.3$, at $z=0$ (e.g., Bullock et al. 2001). 
Plotting SDH density profiles on a logarithmic plane, $(\mathrm{O} \log \eta \log \psi)$, necessarily implies use of dimensionless coordinates, defined as:

$$
\eta=\frac{r}{r_{v i r}} ; \quad \psi(\eta)=\frac{\rho}{\rho_{h}} ; \quad \rho_{h}=\rho_{\text {crit }} \Omega_{m} ;
$$

where an upper and lower limit to the domain follow from the definition of virial radius and the occurrence of numerical artifacts, respectively. This is why (i) regions outside the virial radius are still falling in, and then macroscopic kinetic energy has still to be converted into peculiar energy (e.g., Cole \& Lacey 1996; NFW), and (ii) two-body relaxation takes place in the central regions of simulated haloes, within about $0.01 r_{v i r}$ (e.g., Bullock et al. 2001; Fukushige \& Makino 2001, 2003, 2004; Navarro et al. 2004). Accordingly, SDH density profiles within the range:

$$
-2<\log \eta<0 \quad
$$

appear to be closely related to the virialized region.

\subsection{Comparison between GPL and SDH density pro- files}

A comparison between GPL and SDH density profiles necessarily implies that the truncation radius, and the mass enclosed within the truncation isopycnic surface, do coincide with the virial radius and the virial mass, $R=r_{v i r}$ and $M=M_{v i r}$, respectively. Then the combination of Eqs. (4), (12), and (13), yields:

$$
\begin{aligned}
& \frac{\rho_{0}}{\rho_{h}}=\frac{\Delta_{v i r} \xi_{v i r}^{3}}{\nu_{m a s}} ; \\
& \xi_{v i r}=\frac{r_{v i r}}{r_{0}} ;
\end{aligned}
$$

where the scaled virial radius, $\xi_{v i r}$, may be taken as a definition of the concentration (CMV).

Relating scaled GPL density profiles, expressed by Eqs. (2), to scaled SDH density profiles, expressed by Eqs. (13), yields:

$$
\xi=\xi_{v i r} \eta ; \quad f=\frac{\rho_{h}}{\rho_{0}} \psi
$$


where $\xi_{\text {vir }}$ is defined by Eq. (16). Accordingly, a generic, scaled GPL density profile, expressed by Eq. (3), takes the equivalent form:

$$
\psi(\eta)=\frac{\rho_{0} / \rho_{h}}{\left(\xi_{\text {vir }} \eta\right)^{\gamma}\left[1+\left(\xi_{\text {vir }} \eta\right)^{\alpha}\right]^{\chi}} \quad ; \quad \chi=\frac{\beta-\gamma}{\alpha} ;
$$

and the related, logarithmic GPL density profile, is deduced by use of Eq. (15). The result is:

$$
\begin{aligned}
& \log \psi=\log \Delta_{v i r}-\log \nu_{m a s}+3 \log \xi_{v i r} \\
& -\gamma \log \xi_{v i r}-\gamma \log \eta-\chi \log \left[1+\left(\xi_{v i r} \eta\right)^{\alpha}\right]
\end{aligned}
$$

which depends on three exponents, $(\alpha, \beta, \gamma)$, and two scaling parameters, $\left(r_{0}, \rho_{0}\right)$. On the other hand, the scaled mass, $\nu_{\text {mas }}$, is defined by Eq. (5); the virial radius, $r_{v i r}$, is known from the computer run; and the critical overdensity, $\Delta_{v i r}$, is determined by the cosmological model.

In the logarithmic plane, $(\mathrm{O} \log \eta \log \psi)$, Eq. (19) represents a curve where two asymptotes exist:

$$
\begin{aligned}
& \log \psi=\log \Delta_{v i r}-\log \nu_{m a s}+3 \log \xi_{v i r} \\
& -\gamma \log \xi_{v i r}-\gamma \log \eta ; \quad \xi_{v i r} \eta \ll 1 ; \\
& \log \psi=\log \Delta_{v i r}-\log \nu_{m a s}+3 \log \xi_{v i r} \\
& -\beta \log \xi_{v i r}-\beta \log \eta ; \quad \xi_{v i r} \eta \gg 1 ;
\end{aligned}
$$

which meet at the point $(\log \eta, \log \psi)=\left[\log \left(r_{0} / r_{v i r}\right), \log \left(\rho_{0} / \rho_{h}\right)\right]$. For further details, see CMV.

\subsection{Analytical and geometrical properties of GPL den- sity profiles}

The change of coordinates:

$$
x=\log \eta ; \quad y=\log \psi ;
$$

makes the expressions of the curve and the asymptotes, defined by Eqs. (19), (20a), and (20b), translate into:

$$
\begin{aligned}
& y=y_{C}-\gamma\left(x-x_{C}\right)-\chi \log \left\{1+\exp _{10}\left[\alpha\left(x-x_{C}\right)\right]\right\} ; \\
& y=y_{C}-\gamma\left(x-x_{C}\right) ; \quad x \ll x_{C} ; \\
& y=y_{C}-\beta\left(x-x_{C}\right) ; \quad x \gg x_{C} ;
\end{aligned}
$$


where, in general, $\exp _{u} x=u^{x}$, and $\exp x=\mathrm{e}^{x}$, according to the standard notation. The point, $\mathrm{C}\left(x_{C}, y_{C}\right)$, represents the intersection of the asymptotes in the plane $(\mathrm{O} x y)$, and the expressions of the coordinates are (CMV):

$$
\begin{aligned}
& x_{C}=-\log \xi_{v i r}=-\log \frac{r_{v i r}}{r_{0}}=\log \frac{r_{0}}{r_{v i r}} ; \\
& y_{C}=\log \frac{\Delta_{v i r} \xi_{v i r}^{3}}{\nu_{\text {mas }}}=\log \frac{\rho_{0}}{\rho_{h}} ;
\end{aligned}
$$

which allow the following expression of the scaled mass:

$$
\log \nu_{\text {mas }}=\log \Delta_{v i r}-3 x_{C}-y_{C} .
$$

The vertical intercept of the curve, $b$, and the asymptotes, $b_{\gamma}$ and $b_{\beta}$, may be determined through the combination of Eqs. (22), (23), (24), and (27). The result is:

$$
\begin{aligned}
& b=y_{C}+\gamma x_{C}-\chi \log \left[1+\exp _{10}\left(-\alpha x_{C}\right)\right] ; \\
& b_{\gamma}=y_{C}+\gamma x_{C} ; \\
& b_{\beta}=y_{C}+\beta x_{C}
\end{aligned}
$$

for further details, see CMV.

It can be proved (CMV) that for arbitrarily chosen SPH density profile, cosmological model, and GPL density profile, there is a one-to-one correspondence between analytical parameters, $\left(r_{0}, \rho_{0}, \alpha, \beta, \gamma\right)$, and geometrical parameters, $\left(x_{C}, y_{C}, b, b_{\beta}, b_{\gamma}\right)$, in the sense that either set univocally determines a GPL density profile. Owing to Eqs. (25), and (26), the set $\left(x_{C}, y_{C}, \alpha, \beta, \gamma\right)$ may also be used to this respect.

\section{An analytical RFSM5 method}

Let $\left(x_{i}, y_{i}\right)$ be coordinates of a generic point of a logarithmic SDH density profile, and $\left[x_{i}, y\left(x_{i}\right)\right]$ be their counterparts related to a fitting, GPL density profile. Owing to Eq. (22), the corresponding, logarithmic absolute residual, is:

$$
R_{i}=\left(y_{i}-y_{C}\right)+\gamma\left(x_{i}-x_{C}\right)+\frac{\beta-\gamma}{\alpha} \log \left\{1+\exp _{10}\left[\alpha\left(x_{i}-x_{C}\right)\right]\right\}
$$


where the explicit expression of the exponent, $\chi$, has been used, according to Eq. (1).

With regard to a SDH density profile, a RFSM5 (Residual Function Sum Minimization within a 5-dimension hyperspace) method consists in the minimization of the sum - over the range defined by Eq. (14) - of an assigned residual function (e.g., square, $R^{2}$, or absolute value, $\left.|R|\right)$, within a selected domain of a 5-dimension hyperspace, $\left(\mathrm{O} x_{C} y_{C} \alpha \beta \gamma\right)$, or any equivalent choice of parameters. A numerical procedure has been used in CMV, where the sum of absolute values and squares of residuals was performed on $10^{5}$ nodes of a hypergrid within the above mentioned 5-dimension hyperspace. On the contrary, an analytical procedure shall be exploited here.

In general, let $v=F\left(u_{1}, u_{2}, \ldots, u_{N}\right)$ be a function which is defined in a domain of a $N$-dimension hyperspace, $\left(\mathrm{O} u_{1} u_{2} \ldots u_{N}\right)$, and continuous therein. Then a necessary and sufficient condition for a point, $\mathrm{P}^{*}\left(u_{1}^{*}, u_{2}^{*}, \ldots, u_{N}^{*}\right)$, belonging to the domain, to be an extremal mimimum, is that the coordinates of the point under discussion make (i) a solution of the system, where all the first partial derivatives equal zero:

$$
\left(\frac{\partial F}{\partial u_{j}}\right)_{\mathbf{P}^{*}}=0 \quad ; \quad 1 \leq j \leq N
$$

and (ii) the Hessian determinant positive:

$$
\left|\left(\frac{\partial^{2} F}{\partial u_{j} \partial u_{k}}\right)_{\mathrm{P}^{*}}\right|>0 ; \quad 1 \leq j \leq N \quad ; \quad 1 \leq k \leq N
$$

in the more general case where at least one first partial derivative, $\left(\partial F / \partial u_{j}\right)_{\mathrm{P}^{*}}$, does not exist, Eq. (32) makes a necessary condition for a point, $\mathrm{P}^{*}\left(u_{1}^{*}, u_{2}^{*}, \ldots\right.$, $u_{N}^{*}$ ), to be an extremum point of minimum. For further details see e.g., Smirnov (1969, Chap. V, $\S \S 22-26)$.

Owing to the discontinuity of the first derivative of the absolute value, for null arguments, which makes a solution of the problem much more difficult, our attention shall be limited to the sum of square residuals in the 5-dimension hyperspace of interest:

$$
F\left(x_{C}, y_{C}, \alpha, \beta, \gamma\right)=\sum_{i=1}^{n} R_{i}^{2}
$$


where the $i$-th residual, $R_{i}$, is defined by Eq. (31). Accordingly, Eq. (32) reduces to:

$$
\left(\frac{\partial F}{\partial u_{j}}\right)_{\mathrm{P}^{*}}=2 \sum_{i=1}^{n}\left(R_{i} \frac{\partial R_{i}}{\partial u_{j}}\right)_{\mathrm{P}^{*}}=0 ; \quad 1 \leq j \leq 5
$$

where $u_{1}=x_{C}, u_{2}=y_{C}, u_{3}=\alpha, u_{4}=\beta, u_{5}=\gamma$.

The combination of Eqs. (31), (34), and (35), where the first derivatives are written explicitly, after long but stimulating algebra yields three alternative expressions of the exponent, $\gamma$, as:

$$
\begin{aligned}
& \gamma=\gamma_{1}\left(x_{C}, \alpha\right)=-\frac{v_{s}(x, s) v_{s}(y, t)-v_{s}(s, t) v_{s}(x, y)}{v_{s}(x, s) v_{s}(x, t)-v_{s}(s, t) v_{s}(x, x)} \\
& \gamma=\gamma_{2}\left(x_{C}, \alpha\right)=-\frac{v_{s}(x, s) v_{s}(y, s)-v_{s}(s, s) v_{s}(x, y)}{v_{s}(x, s) v_{s}(x, s)-v_{s}(s, s) v_{s}(x, x)} ; \\
& \gamma=\gamma_{3}\left(x_{C}, \alpha\right)=-\frac{v_{s}(x, s) v_{s}(y, x t)-v_{s}(s, x t) v_{s}(x, y)}{v_{s}(x, s) v_{s}(x, x t)-v_{s}(s, x t) v_{s}(x, x)}
\end{aligned}
$$

where, in general:

$$
\begin{aligned}
& v_{s}(a, b)=\overline{a b}-\bar{a} \bar{b} ; \\
& \bar{c}=\frac{1}{n} \sum_{i=1}^{n} c_{i} ; \quad c=a, b, a b ;
\end{aligned}
$$

and the remaining variables, $s_{i}$ and $t_{i}$, are defined as:

$$
\begin{aligned}
s_{i} & =\log \left(1+w_{i}\right) ; \\
t_{i} & =\frac{w_{i}}{1+w_{i}} ; \\
w_{i} & =\exp _{10}\left[\alpha\left(x_{i}-x_{C}\right)\right] ;
\end{aligned}
$$

where, in addition:

$$
\begin{aligned}
& \frac{\partial w_{i}}{\partial \alpha}=w_{i}\left(x_{i}-x_{C}\right) \ln 10 ; \\
& \frac{\partial w_{i}}{\partial x_{C}}=-w_{i} \alpha \ln 10 ;
\end{aligned}
$$

for a formal demonstration, see Appendix A. 
In a 3-dimension space, $\left(\mathrm{O} x_{C} \alpha \gamma\right)$, Eqs. (36) represent three surfaces. Let $\left(x_{C}^{*} \alpha^{*} \gamma^{*}\right)$ be an intersection point of the above mentioned surfaces. The remaining parameters, $\beta^{*}$ and $y_{C}^{*}$, may be calculated using Eqs. (61) and (59), respectively. The resulting point in a 5-dimension space, $\left(\mathrm{O} x_{C} y_{C} \alpha \beta \gamma\right)$, let it be $\mathrm{P}^{*} \equiv\left(x_{C}^{*}, y_{C}^{*}, \alpha^{*}, \beta^{*}, \gamma^{*}\right)$, satisfies the necessary condition for the occurrence of an extremal minimum, expressed by Eqs. (48). Then a key role is played by the intersections of the above mentioned, three surfaces.

To this regard, the following algorithm is used. First two pairs of surfaces are considered, let they be $\left(\gamma_{1}, \gamma_{2}\right)$ and $\left(\gamma_{1}, \gamma_{3}\right)$, then the intersecting lines related to each pair are simultaneously determined. If the intersecting lines exhibit an intersection point, then the necessary condition for the occurrence of an extremal minimum is satisfied at that point. In the meantime, it can be numerically tested if the intersection point is, in fact, a minimum, thus avoiding the calculation of the Hessian determinant. On the contrary, nothing can be said about the existence of other extremal minima, and the coincidence of the point under consideration with an absolute minimum.

Finding no intersection point does not necessarily mean absence of extremal minima: it could imply that the algorithm used here is insufficient to this respect. If it is the case, a non extremal minimum is selected in the subdomain where the algorithm works.

\section{An application to SDHs on the scale of cluster of galaxies}

Using an analytical RFSM5 method, GPL density profiles are fitted to a sample of $17 \mathrm{SDH}$ density profiles, on the scale of cluster of galaxies within a flat $\Lambda$ CDM cosmology (Rasia et al. 2004), as already done in CMV. The values of the cosmological parameters used therein are: $\Omega_{\Lambda}=0.7 ; \Omega_{m}=0.3$; $\Omega_{b}=0.03 ; h=0.7 ; \sigma_{8}=0.9$; where the symbols have their usual captions (e.g., Klypin et al. 2001; Bullock et al. 2001) and, in particular, the indices $m$ and $b$ denote all matter (dark + baryons) and baryons only, respectively. For a detailed discussion on the computer code, initial conditions, resolution issues, and the way of finding the halo centre, see Rasia et al. (2004) and further references therein.

Simulations include both dark and baryonic matter, but only the former 
is relevant to the aim of the current paper. Accordingly, the baryonic matter shall not be considered, and all the parameters shall be intended as related to the dark matter halo.

The definition of the virialized region within each halo, via Eq. (12), needs the knowledge of the critical overdensity at virialization, $\Delta_{v i r}$. With regard to total (dark + baryonic) matter, it depends on the cosmological model (e.g., Bullock et al. 2001) and, in the case under discussion, $\left(\Delta_{v i r}\right)_{m}=323$ at $z=0$, where all the sample haloes may safely be thought of as virialized (Rasia et al. 2004).

If only the dark matter is considered, then $\left(\Delta_{v i r}\right)_{c d m}=\Delta_{v i r}=\zeta\left(\Delta_{v i r}\right)_{m}$, where $\zeta$ is the fraction of dark matter in each density perturbation, and averaging over the whole sample yields $\zeta=0.907$ (Rasia et al. 2004). Accordingly, the value:

$$
\Delta_{\text {vir }}=0.907 \cdot 323=292.961
$$

can be used to an acceptable extent ${ }^{2}$.

Owing to the random criterion used for the selection (Rasia et al. 2004), sample haloes are characterized by varying dynamical properties: at the present time, some are more relaxed, while others are dynamically perturbed. The surrounding environment can also be quite different: some clusters are more isolated, while others are interacting with the surrounding cosmic web. Accordingly, the related results shall be representative of an averaged cluster, in an averaged environment and dynamical configuration.

\subsection{SDH density profiles}

The main features of sample haloes at $z=0$, are listed in Tab. 1 . The mass, $M_{v i r}$, has been taken from Rasia et al. 2004, while the mass, $M_{v i r}^{\prime}$, has been deduced from Eq. (12). The apparent discrepancy between $M_{v i r}$ and $M_{v i r}^{\prime}$ is owing to two different sources. First, a systematic contribution takes origin from the uncertainty on $\Delta_{v i r}$ and, second, a random contribution arises from

\footnotetext{
${ }^{2}$ The above value of the critical overdensity was deduced from an earlier, unpublished version of Rasia et al. (2004). It is slightly different from $\Delta_{v i r}=0.903 \times 323.7625=$ 292.3576 deduced from the current version, which appeared when all the calculations of this paper were performed. As the relative difference amounts to about $0.2 \%$, the calculations were not repeated using the latter value of the critical overdensity.
} 


\begin{tabular}{cccrrrr}
\hline \hline case & run & type & \multicolumn{1}{c}{$N$} & $r_{\text {vir }}$ & $M_{\text {vir }}$ & $M_{\text {vir }}^{\prime}$ \\
\hline 1 & $S 01.02$ & $R$ & 282574 & 1953 & 76330 & 76040 \\
2 & $S 02.10$ & $M$ & 278569 & 2305 & 125400 & 125010 \\
3 & $S 02.11$ & $M$ & 85159 & 1553 & 38340 & 38234 \\
4 & $S 03.05$ & $R$ & 294373 & 2347 & 132500 & 131970 \\
5 & $S 04.01$ & $R$ & 179681 & 1991 & 80820 & 80565 \\
6 & $S 04.07$ & $R$ & 146386 & 1860 & 65850 & 65686 \\
7 & $S 05.02$ & $M$ & 318653 & 2197 & 108700 & 108249 \\
8 & $S 06.01$ & $M$ & 427583 & 2470 & 153800 & 153825 \\
9 & $S 06.03$ & $M$ & 166855 & 1796 & 60020 & 59136 \\
10 & $S 07.01$ & $R$ & 275259 & 1691 & 49600 & 49359 \\
11 & $S 07.03$ & $R$ & 158345 & 1407 & 28530 & 28433 \\
12 & $S 08.01$ & $R$ & 190453 & 1884 & 68600 & 68262 \\
13 & $S 08.04$ & $R$ & 101482 & 1529 & 36560 & 36489 \\
14 & $S 09.03$ & $R$ & 159330 & 1913 & 71690 & 71463 \\
15 & $S 09.14$ & $R$ & 107229 & 1675 & 48250 & 47971 \\
16 & $S 10.03$ & $R$ & 58734 & 1524 & 36060 & 36132 \\
17 & $S 10.07$ & $R$ & 71937 & 1628 & 44170 & 44045 \\
\hline
\end{tabular}

Table 1: Main features of sample haloes at $z=0$. Column captions: 1 - case; 2 - computer run; 3 - type ( $R$ - safely relaxed; $M$ - safely a major merger occurring); 4 - number of dark matter particles within the virial radius; 5 virial radius $\left(h^{-1} \mathrm{kpc}\right) ; 6$ - virial mass $\left(h^{-1} 10^{10} \mathrm{~m}_{\odot}\right) ; 7$ - virial mass deduced from Eq. (12). Both virial radii and virial masses are normalized to the dimensionless Hubble parameter at the current time, $h$. 
the uncertainty on $r_{v i r}$. An additional random contribution is related to averaging the fraction of dark matter over the whole sample, with regard to Eq. (12).

The relative difference, $\left|1-M_{v i r}^{\prime} / M_{v i r}\right|$, is less then one percent in all cases except 9 , where it is less than one and half percent. The virial mass can be evaluated, to a good extent, by use of Eq. (12), taking the virial radius from the results of simulations. It is worth mentioning that the RFSM5 method is independent of the value of the virial mass, while a change in virial radius makes SDH density profiles systematically shift along the horizontal axis in the logarithmic plane $(\mathrm{O} \log \eta \log \psi)$.

The mean SDH density profile is obtained by averaging over the whole set the values related to each logarithmic radial bin, in the range of interest, expressed by Eq. (14). The value of the critical overdensity at virialization, $\Delta_{v i r}$, is fixed by Eq. (12), then a single free parameter remains: the virial radius, $r_{v i r}$, which allows the calculation of the virial mass, $M_{v i r}$. To this aim, first the virial radius is averaged over the whole sample, using the data listed in Tab. 1, and then the mean value of the virial radius is inserted into Eq. (12). The result is:

$$
\bar{r}_{v i r}=1866 h^{-1} \mathrm{kpc} ; \quad \bar{M}_{v i r}=66330 h^{-1} 10^{10} \mathrm{~m}_{\odot} ;
$$

and the application of a RFSM5 method yields best fitting GPL density profiles, with radius and mass equal to $\bar{r}_{v i r}$ and $\bar{M}_{v i r}$, respectively.

A mean virial radius has been preferred in place of a mean concentration (Rasia et al. 2004) for the following reasons. First, virial radii are independent of GPL density profiles, contrary to concentrations, or velocity profiles, which should be calculated for any choice of the fitting profile. Second, the range in virial radius, $1407 \leq r_{v i r} /\left(h^{-1} \mathrm{kpc}\right) \leq 2470$, corresponds to relative errors of about $25 \%$ and $33 \%$, respectively, with regard to a mean value, $\bar{r}_{v i r}=1866 \mathrm{~h}^{-1} \mathrm{kpc}$. On the other hand, the range in concentration (calculated for NFW density profiles), $5 \leq \xi_{v i r} \leq 10$, corresponds to relative errors of about $32 \%$ and $37 \%$, respectively, with regard to a mean value, $\bar{\xi}_{v i r}=7.2976$. Then the average of the virial radius should be preferred to this respect.

Having in our hands a sample of SDH density profiles, listed in Tab. 1, together with the averaged virial radius, and related virial mass, expressed by Eqs. (41), we are left with the search of a best fitting GPL density profile, 
which minimizes the sum of square residuals. To this aim, the analytical RFSM5 method formulated in section 3, shall be used.

\subsection{Results}

As outlined in section 3, an analytical RFSM5 method has been applied to each sample halo, listed in Tab. 1, and to the related, mean SDH density profile, which has been defined above. The values of the exponents, $\alpha, \chi, \beta$, $\gamma$, the scaled radius, $\xi_{\text {vir }}$, the scaling radius, $r_{0}$, the scaling density, $\rho_{0}$, and the sum of absolute value residuals, $\sum\left|R_{i}\right|$, and square residuals, $\sum R_{i}^{2}$, at the extremal or fiducial minimum, are listed in Tabs. 2 and 3, respectively, for both analytical (current paper, upper lines) and numerical (CMV, lower lines) RFSM5 methods. The following conclusions are deduced.

(i) A necessary condition for the occurrence of an extremal point, expressed by Eqs. (36), is satisfied for $8 \mathrm{SDHs}$ and is not for the remaining 9 together with ADP. In the former alternative, an extremal minimum point may safely exist. In the latter alternative, the existence of an extremal minimum point cannot be established, but only a non extremal minimum may be determined. This last is related to the GPL density profile where the distance between intersecting lines of two distinct pairs of surfaces, defined by Eqs. (36), attains the lowest value. The above mentioned, non extremal minimum, may be located both within (IMP) and on the boundary (BMP) of the subdomain where the RFSM5 method, used in the current paper, works.

(ii) The sum of square residuals is systematically lower using analytical (current paper) instead of numerical (CMV) RFSM5 methods, provided an extremal minimum point occurs. Accordingly, EMPs may safely be thought of as absolute minima. With regard to non extremal minimum points, the above result maintains in 4 cases (including ADP) while the contrary holds for the remaining 5 cases. The trend is not necessarily the same for the sum of absolute value residuals, as expected.

(iii) The exponents, $\alpha, \beta, \gamma$, range within $0.4<\alpha<19.8(\alpha=0.2900$ for ADP); $1.9<\beta<17.6 ; 0.6<\gamma<1.8$; and $0.4<\alpha<0.9$; $2.5<\beta<3.9 ; 0.7<\gamma<1.3$; using analytical and numerical RFSM5 methods, respectively. With regard to SDH density profiles where 


\begin{tabular}{|c|c|c|c|c|c|c|}
\hline case & type & $\bar{\alpha}$ & $\bar{\chi}$ & $\bar{\beta}$ & $\gamma$ & class \\
\hline \multirow[t]{2}{*}{1} & $\mathrm{I}$ & 0.76000 & 21.296 & 17.533 & 1.3480 & IMP \\
\hline & & 0.85979 & 2.7291 & 3.4893 & 1.1429 & \\
\hline \multirow[t]{2}{*}{2} & M & 8.96000 & 0.081928 & 2.1484 & 1.4143 & BMP \\
\hline & & 0.70267 & 2.6771 & 2.8238 & 0.94262 & \\
\hline \multirow[t]{2}{*}{3} & M & 7.60000 & 0.072676 & 2.1063 & 1.5540 & IMP \\
\hline & & 0.50594 & 3.6824 & 3.0631 & 1.2000 & \\
\hline \multirow[t]{2}{*}{4} & $\mathrm{I}$ & 3.9760 & 0.14895 & 1.9593 & 1.3671 & IMP \\
\hline & & 0.40157 & 4.8960 & 2.9564 & 0.99023 & \\
\hline \multirow[t]{2}{*}{5} & I & 5.4644 & 0.12476 & 2.3843 & 1.7026 & EMP \\
\hline & & 0.59295 & 3.2455 & 3.1244 & 1.2000 & \\
\hline \multirow[t]{2}{*}{6} & $\mathrm{I}$ & 19.787 & 0.027126 & 2.0238 & 1.4870 & EMP \\
\hline & & 0.50437 & 2.8516 & 2.5152 & 1.0769 & \\
\hline \multirow[t]{2}{*}{7} & M & 7.5000 & 0.13191 & 2.2709 & 1.2816 & IMP \\
\hline & & 0.60451 & 4.1915 & 3.5715 & 1.0377 & \\
\hline \multirow[t]{2}{*}{8} & M & 0.42000 & 12.214 & 5.7327 & 0.60284 & IMP \\
\hline & & 0.66861 & 3.9138 & 3.4748 & 0.85806 & \\
\hline \multirow[t]{2}{*}{9} & M & 0.77830 & 2.1159 & 2.7751 & 1.1283 & EMP \\
\hline & & 0.58477 & 3.7717 & 3.2083 & 1.0027 & \\
\hline \multirow[t]{2}{*}{10} & I & 13.5000 & 0.045375 & 1.9470 & 1.3344 & IMP \\
\hline & & 0.43019 & 3.7611 & 2.7372 & 1.1192 & \\
\hline \multirow[t]{2}{*}{11} & I & 3.3124 & 0.28130 & 2.5344 & 1.6026 & EMP \\
\hline & & 0.69931 & 3.3055 & 3.4625 & 1.1509 & \\
\hline \multirow[t]{2}{*}{12} & I & 2.9758 & 0.35736 & 2.0978 & 1.0344 & EMP \\
\hline & & 0.67451 & 3.0786 & 2.7795 & 0.70312 & \\
\hline \multirow[t]{2}{*}{13} & I & 2.1590 & 0.53250 & 2.5151 & 1.3655 & EMP \\
\hline & & 0.68562 & 3.3840 & 3.1595 & 0.83933 & \\
\hline \multirow[t]{2}{*}{14} & I & 0.57290 & 6.0145 & 4.3163 & 0.87060 & EMP \\
\hline & & 0.81937 & 2.4907 & 2.9870 & 0.94621 & \\
\hline \multirow[t]{2}{*}{15} & I & 3.8132 & 0.22865 & 2.2556 & 1.3837 & EMP \\
\hline & & 0.70472 & 2.8497 & 2.8694 & 0.86117 & \\
\hline \multirow[t]{2}{*}{16} & I & 1.4000 & 1.2555 & 3.0649 & 1.3072 & IMP \\
\hline & & 0.74414 & 3.9381 & 3.8674 & 0.93684 & \\
\hline \multirow[t]{2}{*}{17} & I & 11.0000 & 0.039069 & 1.9504 & 1.5207 & IMP \\
\hline & & 0.44450 & 4.0781 & 2.8351 & 1.0224 & \\
\hline \multirow[t]{2}{*}{$\mathrm{ADP}$} & & 0.29000 & 14.846 & 4.4590 & 1.5360 & IMP \\
\hline & & 0.56832 & 4.0722 & 3.3143 & 1.0000 & \\
\hline
\end{tabular}

Table 2: Exponents of GPL density profiles which minimize the sum of square residuals, using (i) an analytical RFSM5 method (current paper, upper lines), and (ii) a numerical RFSM5 method (CMV, lower lines), with regard to 17 sample haloes listed in Tab. 1, together with the averaged density profile (ADP). Type captions: I - safely relaxed; M - safely a major merger occurring. Class captions: EMP - extremal minimum point; IMP - minimum point occurring within the subdomain interested by the application of the method, 


\begin{tabular}{|c|c|c|c|c|c|c|c|}
\hline case & type & $\overline{\overline{\xi_{v i r}}}$ & $r_{0}$ & $\rho_{0}$ & 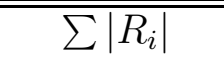 & $\overline{\sum R_{i}^{2}}$ & class \\
\hline \multirow[t]{2}{*}{1} & I & $8.5258 \mathrm{E}-2$ & $3.2724 \mathrm{E}+4$ & $1.4808 \mathrm{E}-7$ & $2.7556 \mathrm{E}-1$ & $5.3397 \mathrm{E}-3$ & IMP \\
\hline & & $3.6308 \mathrm{E}+0$ & $7.6843 \mathrm{E}+2$ & $4.5733 \mathrm{E}-5$ & $4.3189 \mathrm{E}-1$ & $1.2843 \mathrm{E}-2$ & \\
\hline \multirow[t]{2}{*}{2} & M & $1.0235 \mathrm{E}+1$ & $3.2172 \mathrm{E}+2$ & $5.1755 \mathrm{E}-5$ & $6.4689 \mathrm{E}-1$ & $3.5769 \mathrm{E}-2$ & BMP \\
\hline & & $7.1450 \mathrm{E}+0$ & $4.6086 \mathrm{E}+2$ & $1.4462 \mathrm{E}-4$ & $6.7590 \mathrm{E}-1$ & $5.0984 \mathrm{E}-2$ & \\
\hline \multirow[t]{2}{*}{3} & M & $2.0099 \mathrm{E}+1$ & $1.1038 \mathrm{E}+3$ & $2.0053 \mathrm{E}-4$ & $6.9707 \mathrm{E}-1$ & $7.5840 \mathrm{E}-2$ & IMP \\
\hline & & $4.4668 \mathrm{E}+0$ & $4.9668 \mathrm{E}+2$ & $1.1755 \mathrm{E}-4$ & $8.2813 \mathrm{E}-1$ & $5.9798 \mathrm{E}-2$ & \\
\hline \multirow[t]{2}{*}{4} & I & $2.3393 \mathrm{E}+1$ & $1.4333 \mathrm{E}+2$ & $2.0029 \mathrm{E}-4$ & $7.3567 \mathrm{E}-1$ & $5.1734 \mathrm{E}-2$ & IMP \\
\hline & & $4.1115 \mathrm{E}+0$ & $8.1548 \mathrm{E}+2$ & $2.1391 \mathrm{E}-4$ & $7.7711 \mathrm{E}-1$ & $4.4834 \mathrm{E}-2$ & \\
\hline \multirow[t]{2}{*}{5} & $\mathrm{I}$ & $7.0536 \mathrm{E}+0$ & $4.0324 \mathrm{E}+2$ & $3.0094 \mathrm{E}-5$ & $3.9790 \mathrm{E}-1$ & $1.3080 \mathrm{E}-2$ & EMP \\
\hline & & $4.7863 \mathrm{E}+0$ & $5.9426 \mathrm{E}+2$ & $1.0721 \mathrm{E}-4$ & $4.5994 \mathrm{E}-1$ & $1.5935 \mathrm{E}-2$ & \\
\hline \multirow[t]{2}{*}{6} & $\mathrm{I}$ & $2.0441 \mathrm{E}+1$ & $1.2999 \mathrm{E}+2$ & $1.7204 \mathrm{E}-4$ & $3.7559 \mathrm{E}-1$ & $1.2647 \mathrm{E}-2$ & EMP \\
\hline & & $1.6501 \mathrm{E}+1$ & $1.6103 \mathrm{E}+2$ & $7.3632 \mathrm{E}-4$ & $6.2224 \mathrm{E}-1$ & $3.3055 \mathrm{E}-2$ & \\
\hline \multirow[t]{2}{*}{7} & M & $2.1719 \mathrm{E}+1$ & $1.4451 \mathrm{E}+2$ & $3.3786 \mathrm{E}-4$ & $7.9368 \mathrm{E}-1$ & $4.8776 \mathrm{E}-2$ & IMP \\
\hline & & $4.2267 \mathrm{E}+0$ & $7.4256 \mathrm{E}+2$ & $1.7070 \mathrm{E}-4$ & $6.1250 \mathrm{E}-1$ & $2.9137 \mathrm{E}-2$ & \\
\hline \multirow[t]{2}{*}{8} & M & $5.4741 \mathrm{E}-1$ & $6.4459 \mathrm{E}+3$ & $1.9954 \mathrm{E}-4$ & $2.4863 \mathrm{E}-1$ & $4.5390 \mathrm{E}-3$ & IMP \\
\hline & & $4.1687 \mathrm{E}+0$ & $8.4645 \mathrm{E}+2$ & $1.3190 \mathrm{E}-4$ & $2.2446 \mathrm{E}-1$ & $4.9367 \mathrm{E}-3$ & \\
\hline \multirow[t]{2}{*}{9} & M & $9.4211 \mathrm{E}+0$ & $2.7234 \mathrm{E}+2$ & $2.0100 \mathrm{E}-4$ & $2.6901 \mathrm{E}-1$ & $5.7462 \mathrm{E}-3$ & EMP \\
\hline & & $5.6234 \mathrm{E}+0$ & $4.5626 \mathrm{E}+2$ & $2.2399 \mathrm{E}-4$ & $2.8092 \mathrm{E}-1$ & $6.1089 \mathrm{E}-3$ & \\
\hline \multirow[t]{2}{*}{10} & I & $3.3231 \mathrm{E}+1$ & $7.2696 \mathrm{E}+2$ & $3.8804 \mathrm{E}-4$ & $4.6024 \mathrm{E}-1$ & $2.4169 \mathrm{E}-2$ & IMP \\
\hline & & $6.6374 \mathrm{E}+0$ & $3.6395 \mathrm{E}+2$ & $2.5132 \mathrm{E}-4$ & $5.0765 \mathrm{E}-1$ & $2.0731 \mathrm{E}-2$ & \\
\hline \multirow[t]{2}{*}{11} & $\mathrm{I}$ & $6.9351 \mathrm{E}+0$ & $2.8983 \mathrm{E}+2$ & $3.4633 \mathrm{E}-5$ & $3.3590 \mathrm{E}-1$ & $9.4062 \mathrm{E}-3$ & EMP \\
\hline & & $3.7154 \mathrm{E}+0$ & $5.4100 \mathrm{E}+2$ & $6.7645 \mathrm{E}-5$ & $4.5841 \mathrm{E}-1$ & $1.7674 \mathrm{E}-2$ & \\
\hline \multirow[t]{2}{*}{12} & $\mathrm{I}$ & $1.6379 \mathrm{E}+1$ & $1.6432 \mathrm{E}+2$ & $1.2941 \mathrm{E}-4$ & $4.8093 \mathrm{E}-1$ & $2.8344 \mathrm{E}-2$ & EMP \\
\hline & & $9.1201 \mathrm{E}+0$ & $3.6319 \mathrm{E}+2$ & $2.8856 \mathrm{E}-4$ & $8.1443 \mathrm{E}-1$ & $5.0487 \mathrm{E}-2$ & \\
\hline \multirow[t]{2}{*}{13} & $\mathrm{I}$ & $8.0514 \mathrm{E}+0$ & $2.4964 \mathrm{E}+2$ & $5.2008 \mathrm{E}-5$ & $6.1515 \mathrm{E}-1$ & $3.2976 \mathrm{E}-2$ & EMP \\
\hline & & $6.8234 \mathrm{E}+0$ & $3.2012 \mathrm{E}+2$ & $2.5718 \mathrm{E}-4$ & $5.9097 \mathrm{E}-1$ & $3.6570 \mathrm{E}-2$ & \\
\hline \multirow[t]{2}{*}{14} & I & $1.3437 \mathrm{E}+0$ & $2.0338 \mathrm{E}+3$ & $3.9969 \mathrm{E}-5$ & $3.8299 \mathrm{E}-1$ & $1.1169 \mathrm{E}-2$ & EMP \\
\hline & & $5.3088 \mathrm{E}+0$ & $5.1477 \mathrm{E}+2$ & $7.7666 \mathrm{E}-5$ & $4.1735 \mathrm{E}-1$ & $1.2273 \mathrm{E}-2$ & \\
\hline \multirow[t]{2}{*}{15} & $\mathrm{I}$ & $8.9712 \mathrm{E}+0$ & $2.6673 \mathrm{E}+2$ & $4.6219 \mathrm{E}-5$ & $4.9331 \mathrm{E}-1$ & $2.0331 \mathrm{E}-2$ & EMP \\
\hline & & $7.6913 \mathrm{E}+0$ & $3.1111 \mathrm{E}+2$ & $1.9963 \mathrm{E}-4$ & $6.0948 \mathrm{E}-1$ & $2.8821 \mathrm{E}-2$ & \\
\hline \multirow[t]{2}{*}{16} & I & $5.2999 \mathrm{E}+0$ & $4.1079 \mathrm{E}+2$ & $3.8564 \mathrm{E}-5$ & $6.4586 \mathrm{E}-1$ & $2.9673 \mathrm{E}-2$ & IMP \\
\hline & & $3.7154 \mathrm{E}+0$ & $5.8599 \mathrm{E}+2$ & $1.1226 \mathrm{E}-4$ & $4.4819 \mathrm{E}-1$ & $1.5259 \mathrm{E}-2$ & \\
\hline \multirow[t]{2}{*}{17} & $\mathrm{I}$ & $1.4047 \mathrm{E}+1$ & $1.6556 \mathrm{E}+2$ & $7.2820 \mathrm{E}-5$ & $7.4326 \mathrm{E}-1$ & $7.3645 \mathrm{E}-2$ & IMP \\
\hline & & $4.6774 \mathrm{E}+0$ & $4.9723 \mathrm{E}+2$ & $1.5144 \mathrm{E}-4$ & $7.1010 \mathrm{E}-1$ & $5.7718 \mathrm{E}-2$ & \\
\hline \multirow[t]{2}{*}{$\mathrm{ADP}$} & & $2.2073 \mathrm{E}+0$ & $1.2077 \mathrm{E}+3$ & $5.9044 \mathrm{E}-6$ & 1.1027 E-1 & $1.1783 \mathrm{E}-3$ & IMP \\
\hline & & $3.6308 \mathrm{E}+0$ & $7.3422 \mathrm{E}+2$ & $1.0238 \mathrm{E}-4$ & $1.3262 \mathrm{E}-1$ & $1.4983 \mathrm{E}-3$ & \\
\hline
\end{tabular}

Table 3: Scale parameters of GPL density profiles which minimize the sum of square residuals, using (i) an analytical RFSM5 method (current paper, upper lines), and (ii) a numerical RFSM5 method (CMV, lower lines), with regard to 17 sample haloes listed in Tab. 1, together with the averaged density profile $(\mathrm{ADP})$. The scaling radius, $r_{0}$, and scaling density, $\rho_{0}$, are expressed in $\mathrm{kpc}$ and $10^{10} \mathrm{~m}_{\odot} / \mathrm{kpc}^{3}$, respectively. Other captions as in Tab. 2 
an EMP occurs, the above ranges in the former alternative reduce to $0.5<\alpha<19.8 ; 2.0<\beta<4.4$ ( $\beta=4.4590$ for ADP $) ; 0.8<\gamma<1.8$; which is, only for $\beta$, substantially closer to their counterparts related to the latter alternative. Accordingly, analytical RFSM5 methods yield GPL density profiles where the range in the exponents is larger than in their counterparts related to numerical RSFM5 methods. It provides additional support to the occurrence of a certain degree of degeneracy (e.g., Klypin et al. 2001; CMV).

(iv) The scaled radius, $\xi_{\text {vir }}$, the scaling radius, $r_{0}$, and the scaling density, $\rho_{0}$, range within $0.08<\xi_{v i r}<33.3 ; 72<r_{0} / \mathrm{kpc}<32724$; $0.01<10^{4} \rho_{0} /\left(10^{10} \mathrm{~m}_{\odot} / \mathrm{kpc}^{3}\right)<3.9 ;$ and $3.6<\xi_{\text {vir }}<16.5 ; 161<$ $r_{0} / \mathrm{kpc}<847 ; 0.4<10^{4} \rho_{0} /\left(10^{10} \mathrm{~m}_{\odot} / \mathrm{kpc}^{3}\right)<7.4 ;$ using analytical and numerical RFSM5 methods, respectively. With regard to SDH density profiles where an EMP occurs, the above ranges in the former alternative reduce to $1.3<\xi_{\text {vir }}<20.5 ; 129<r_{0} / \mathrm{kpc}<2034$; $0.3<10^{4} \rho_{0} /\left(10^{10} \mathrm{~m}_{\odot} / \mathrm{kpc}^{3}\right)<2.1$; which is much closer to their counterparts related to the latter alternative.

The occurrence of high values of the exponents, $\alpha$ and $\beta$, related to (a) lack of an EMP, and (b) lower value of the sum of square residuals using analytical instead of numerical RFSM5 methods, as in cases 1 and 10 of Tab. 2, suggests further investigation on the best fitting, limiting GPL density profiles, analysed in subsection 2.1.

According to Eq. (7), GPL density profiles reduce to a double power-law (DPL) with exponents equal to $-\gamma$ and $-\beta$, in the limit $\alpha \rightarrow \infty, \chi \rightarrow 0$, i.e. $\gamma$ and $\beta$ finite. Then we are left with two exponents instead of three, and a RFSM4 method has to be used, within a 4-dimension hyperspace $\left(\mathrm{O} x_{C} y_{C} \beta \gamma\right.$ ), in dealing with each sample halo, listed in Tab.1, together with the related ADP, which has been defined above. The values of the exponents, $\beta$ and $\gamma$, the scaled radius, $\xi_{\text {vir }}$, the scaling radius, $r_{0}$, the scaling density, $\rho_{0}$, the sum of absolute value residuals, $\sum\left|R_{i}\right|$, and the sum of square residuals, $\sum R_{i}^{2}$, are listed in Tab. 4 . By comparison with Tabs. 2 and 3, the following conclusions are deduced.

(v) The sum of square residuals is systematically larger with respect to analytical RFSM5 methods, provided an EMP occurs, as expected. Concerning non extremal minimum points, the above result maintains 


\begin{tabular}{ccccccc}
\hline \hline$\beta$ & $\gamma$ & $\xi_{\text {vir }}$ & $r_{0}$ & $\rho_{0}$ & $\sum\left|R_{i}\right|$ & $\sum R_{i}^{2}$ \\
\hline 2.8057 & 1.6263 & $3.9799 \mathrm{E}+0$ & $7.1465 \mathrm{E}+2$ & $1.0852 \mathrm{E}-5$ & $9.9122 \mathrm{E}-1$ & $1.4472 \mathrm{E}-1$ \\
2.1545 & 1.4338 & $9.6963 \mathrm{E}+0$ & $2.9334 \mathrm{E}+2$ & $4.8193 \mathrm{E}-5$ & $6.4395 \mathrm{E}-1$ & $3.5625 \mathrm{E}-2$ \\
3.4200 & 1.8638 & $1.9336 \mathrm{E}+0$ & $1.4710 \mathrm{E}+3$ & $1.9761 \mathrm{E}-6$ & $9.1288 \mathrm{E}-1$ & $5.1722 \mathrm{E}-2$ \\
3.0400 & 1.7367 & $1.9743 \mathrm{E}+0$ & $1.4407 \mathrm{E}+3$ & $2.0941 \mathrm{E}-6$ & $7.0852 \mathrm{E}-1$ & $4.3206 \mathrm{E}-2$ \\
2.3798 & 1.7257 & $6.6950 \mathrm{E}+0$ & $4.2484 \mathrm{E}+2$ & $2.7050 \mathrm{E}-5$ & $3.9284 \mathrm{E}-1$ & $1.3590 \mathrm{E}-2$ \\
2.0238 & 1.4900 & $2.0314 \mathrm{E}+1$ & $1.4001 \mathrm{E}+2$ & $1.7556 \mathrm{E}-4$ & $3.7440 \mathrm{E}-1$ & $1.2651 \mathrm{E}-2$ \\
2.2659 & 1.3214 & $2.0573 \mathrm{E}+1$ & $1.3825 \mathrm{E}+2$ & $2.9438 \mathrm{E}-4$ & $8.5352 \mathrm{E}-1$ & $5.0746 \mathrm{E}-2$ \\
2.3228 & 1.4364 & $8.1896 \mathrm{E}+0$ & $3.4731 \mathrm{E}+2$ & $4.1276 \mathrm{E}-5$ & $5.6538 \mathrm{E}-1$ & $2.3083 \mathrm{E}-2$ \\
2.3222 & 1.6425 & $8.0737 \mathrm{E}+0$ & $3.5229 \mathrm{E}+2$ & $3.9237 \mathrm{E}-5$ & $4.2369 \mathrm{E}-1$ & $1.4978 \mathrm{E}-2$ \\
1.9452 & 1.3370 & $3.3206 \mathrm{E}+1$ & $8.5655 \mathrm{E}+2$ & $3.8561 \mathrm{E}-4$ & $4.6730 \mathrm{E}-1$ & $2.4272 \mathrm{E}-2$ \\
2.4412 & 1.6360 & $7.5301 \mathrm{E}+0$ & $3.7772 \mathrm{E}+2$ & $3.7991 \mathrm{E}-5$ & $4.0076 \mathrm{E}-1$ & $1.3061 \mathrm{E}-2$ \\
2.0438 & 1.1446 & $1.5593 \mathrm{E}+1$ & $1.8241 \mathrm{E}+2$ & $1.0817 \mathrm{E}-4$ & $6.1143 \mathrm{E}-1$ & $3.3369 \mathrm{E}-2$ \\
2.4638 & 1.5349 & $6.4809 \mathrm{E}+0$ & $4.3887 \mathrm{E}+2$ & $2.7799 \mathrm{E}-5$ & $7.0267 \mathrm{E}-1$ & $3.8464 \mathrm{E}-2$ \\
2.2258 & 1.3844 & $8.2336 \mathrm{E}+0$ & $3.4545 \mathrm{E}+2$ & $3.7441 \mathrm{E}-5$ & $5.1443 \mathrm{E}-1$ & $2.2151 \mathrm{E}-2$ \\
2.2410 & 1.4449 & $8.1530 \mathrm{E}+0$ & $3.4886 \mathrm{E}+2$ & $3.7169 \mathrm{E}-5$ & $5.3382 \mathrm{E}-1$ & $2.2494 \mathrm{E}-2$ \\
2.5215 & 1.5093 & $7.8512 \mathrm{E}+0$ & $3.6228 \mathrm{E}+2$ & $4.6051 \mathrm{E}-5$ & $8.7268 \mathrm{E}-1$ & $5.5935 \mathrm{E}-2$ \\
1.9366 & 1.5001 & $1.5659 \mathrm{E}+1$ & $1.8164 \mathrm{E}+2$ & $8.8556 \mathrm{E}-5$ & $7.1163 \mathrm{E}-1$ & $7.3474 \mathrm{E}-2$ \\
2.1974 & 1.5207 & $9.7371 \mathrm{E}+0$ & $2.9211 \mathrm{E}+2$ & $5.1240 \mathrm{E}-5$ & $4.2918 \mathrm{E}-1$ & $1.3103 \mathrm{E}-2$ \\
\hline \hline
\end{tabular}

Table 4: Parameters of double power-law (DPL) density profiles which minimize the sum of square residuals, using an analytical RFSM4 method, with regard to 17 sample haloes listed in Tab. 1 together with the averaged density profile (ADP). Other captions as in Tabs. 2-3. Cases, types, and classes have been omitted to save space. 
in 6 cases (including ADP), while the contrary holds for the remaining 4 cases. The sum of square residuals is larger, with respect to numerical RSFM5 methods, in 11 cases (including ADP), while the contrary holds for the remaining 8 cases. The trend is not necessarily the same for the sum of absolute value residuals, as expected.

(vi) The exponents, $\beta$ and $\gamma$, range within $1.9<\beta<3.5$ and $1.1<\gamma<$ 1.9 , respectively, which are comparable to their counteparts related to both analytical (restricted to occurring EMPs) and numerical RSFM5 methods.

(vii) The scaled radius, $\xi_{v i r}$, the scaling radius, $r_{0}$, and the scaling density, $\rho_{0}$, range within $1.9<\xi_{\text {vir }}<33.3 ; 85<r_{0} / \mathrm{kpc}<1471$; and $0.01<$ $10^{4} \rho_{0} /\left(10^{10} \mathrm{~m}_{\odot} / \mathrm{kpc}^{3}\right)<3.9$; respectively, which are comparable to their counterparts related to both analytical (restricted to occurring EMPs) and numerical RFSM5 methods.

Then DPL density profiles may be considered as a rough, but viable approximation in fitting SDH density profiles.

According to Eqs. (8) and (9), GPL density profiles reduce to a simple power-law (SPL) with slope equal to $-\gamma$, in the limit $\beta \rightarrow+\infty$ where $\alpha, \gamma$, and $\left(1+\xi^{\alpha}\right)^{\chi}$ remain finite, and $\alpha \rightarrow 0$ where $\beta \rightarrow \gamma$ and $\left(1+\xi^{\alpha}\right)^{\chi} \rightarrow 2^{\chi}$. In any case, we are left with one exponent instead of three and one coordinate (the vertical intercept of SPL density profiles in the logarithmic plane, $b_{\gamma}$ ) instead of two. Then a RFSM2 method i.e. a standard least-squares fit has to be used, within a plane $\left(\mathrm{O} b_{\gamma} \gamma\right)$, in dealing with each sample halo, listed in Tab. 1, together with the related ADP, which has been defined above. The values of the exponent, $\gamma$, the intercept, $b_{\gamma}$, the sum of absolute value residuals, $\sum\left|R_{i}\right|$, and the sum of square residuals, $\sum R_{i}^{2}$, are listed in Tab. 5 . By comparison with Tabs. 2 and 3, the following conclusions are deduced.

(viii) The sum of square residuals is systematically larger with respect to both analytical and numerical RFSM5 methods. The same holds also for the sum of absolute value residuals.

(ix) The exponent, $\gamma$, and the intercept, $b_{\gamma}$, range within $1.7<\gamma<2.1$ and $2.0<b_{\gamma}<2.2$, which is larger and lower, respectively, with regard to their counteparts related to both analytical and numerical RSFM5 methods, as expected. 


\begin{tabular}{cccclll}
\hline \hline case & type & $\gamma$ & $b_{\gamma}$ & $\sum\left|R_{i}\right|$ & \multicolumn{1}{c}{$\sum R_{i}^{2}$} & class \\
\hline 1 & $\mathrm{I}$ & 1.9078 & 2.0722 & 2.2846 & 0.35794 & IMP \\
2 & $\mathrm{M}$ & 1.7869 & 2.1141 & 1.8812 & 0.24998 & BMP \\
3 & $\mathrm{M}$ & 1.9499 & 2.0314 & 1.3315 & 0.16573 & IMP \\
4 & $\mathrm{I}$ & 1.8133 & 2.0893 & 1.2889 & 0.12975 & IMP \\
5 & $\mathrm{I}$ & 1.9680 & 2.0322 & 1.5870 & 0.17503 & EMP \\
6 & $\mathrm{I}$ & 1.8764 & 2.0567 & 1.1596 & 0.099672 & EMP \\
7 & $\mathrm{M}$ & 2.0086 & 2.0223 & 2.0516 & 0.32024 & IMP \\
8 & $\mathrm{M}$ & 1.8220 & 2.1132 & 2.1881 & 0.34027 & IMP \\
9 & $\mathrm{M}$ & 1.9351 & 2.0572 & 1.7520 & 0.20064 & EMP \\
10 & $\mathrm{I}$ & 1.8576 & 2.0682 & 0.96462 & 0.082826 & IMP \\
11 & $\mathrm{I}$ & 1.9644 & 2.0418 & 2.0423 & 0.26923 & EMP \\
12 & $\mathrm{I}$ & 1.7228 & 2.1469 & 2.2995 & 0.33084 & EMP \\
13 & $\mathrm{I}$ & 1.8695 & 2.0913 & 2.3278 & 0.35789 & EMP \\
14 & $\mathrm{I}$ & 1.7519 & 2.1432 & 2.0687 & 0.30842 & EMP \\
15 & $\mathrm{I}$ & 1.7901 & 2.1227 & 2.0813 & 0.27796 & EMP \\
16 & $\mathrm{I}$ & 1.9358 & 2.0614 & 2.5099 & 0.46469 & IMP \\
17 & $\mathrm{I}$ & 1.7813 & 2.0982 & 1.2451 & 0.14339 & IMP \\
ADP & & 1.8531 & 2.0856 & 1.7061 & 0.20209 & IMP \\
\hline \hline
\end{tabular}

Table 5: Parameters of single power-law (SPL) density profiles which minimize the sum of square residuals, using an analytical RFSM2 method i.e. a standard least squares fit, with regard to 17 sample haloes listed in Tab. 1 together with the averaged density profile (ADP). Other captions as in Tabs. 2 and 3 . 
Then SPL density profiles cannot be considered as an acceptable approximation to GPL density profiles, in fitting SDH density profiles.

To get further insight, the sum of square residuals is listed in Tab. 6 for all the four alternatives which have been exploited in the current section. The values of the exponents, $\alpha, \chi, \beta, \gamma$, the scaled radius, $\xi_{\text {vir }}$, the scaling radius, $r_{0}$, the scaling density, $\rho_{0}$, and the sum of absolute value residuals, $\sum\left|R_{i}\right|$, and square residuals, $\sum R_{i}^{2}$, at the extremal or fiducial minimum, are listed in Tabs. 7 and 8, respectively, with regard to the best fit, among the ones considered above, for each sample halo.

The values of some analytical and geometrical parameters, $\eta_{A D P}$, related to the best fitting GPL density profile to the mean SDH density profile, are listed in Tab. 9 together with their counterparts, $\bar{\eta}$, averaged over the best fitting GPL density profiles to the whole halo sample, using the data of Tab. 8. Also listed in Tab. 9 are the related standard deviations, $\sigma_{s} \eta$, the standard deviations from the mean, $\sigma_{s} \bar{\eta}$, and the standard deviations from the standard deviation from the mean, $\sigma_{s} \bar{\mu}$, which are expressed as (e.g., Oliva \& Terrasi 1976, Chap. V, § 5.6.3):

$$
\begin{aligned}
& \bar{\eta}=\frac{1}{n} \sum_{i=1}^{n} \eta_{i} ; \\
& \sigma_{s \eta}=\left[\frac{1}{n-1} \sum_{i=1}^{n}\left(\eta_{i}-\bar{\eta}\right)^{2}\right]^{1 / 2} ; \\
& \sigma_{s \bar{\eta}}=\left[\frac{1}{n} \frac{1}{n-1} \sum_{i=1}^{n}\left(\eta_{i}-\bar{\eta}\right)^{2}\right]^{1 / 2} ; \\
& \sigma_{s \bar{\mu}}=\frac{\sigma_{s \bar{\eta}}}{\sqrt{2 n}} ; \quad \bar{\mu}=\sigma_{s \bar{\eta}} ;
\end{aligned}
$$

where $\eta=\alpha, \beta, \gamma, \xi_{\text {vir }}, \nu_{\text {mas }}, \log \xi_{\text {vir }}, \log \nu_{\text {mas }}, y_{C}, b, b_{\beta}, b_{\gamma} ; n=17$ in all cases but $n=14$ if $\eta=\alpha$, as $\alpha \rightarrow+\infty$, related to DPL density profiles, cannot be considered in averaging; and, owing to Eq. (25), $\log \xi_{v i r}=-x_{C}$.

The following conclusions are deduced from Tab. 9.

(x) Values of parameters, $\eta_{A D P}$, related to the best fitting GPL density profile to the mean SDH density profile, are different from their counterparts averaged over the best fitting GPL density profiles to the whole halo sample, listed in Tabs. 7 and 8. The discrepancy changes for differ- 


\begin{tabular}{|c|c|c|c|c|c|c|}
\hline case & type & $\overline{\overline{\text { GPN }}}$ & $\overline{\overline{\text { GPA }}}$ & 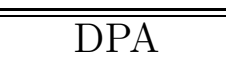 & $\overline{\overline{\text { SPA }}}$ & $\begin{array}{cc}\text { class } \\
\end{array}$ \\
\hline 1 & I & $1.2843 \mathrm{E}-2$ & $5.3397 \mathrm{E}-3$ & $1.4472 \mathrm{E}-1$ & $3.5794 \mathrm{E}-1$ & IMP \\
\hline 2 & M & $5.0984 \mathrm{E}-2$ & $3.5769 \mathrm{E}-2$ & $3.5625 \mathrm{E}-2$ & $2.4998 \mathrm{E}-1$ & BMP \\
\hline 3 & M & $5.9798 \mathrm{E}-2$ & $7.5840 \mathrm{E}-2$ & $5.1722 \mathrm{E}-2$ & $1.6573 \mathrm{E}-1$ & IMP \\
\hline 4 & I & $4.4834 \mathrm{E}-2$ & $5.1734 \mathrm{E}-2$ & $4.3206 \mathrm{E}-2$ & $1.2975 \mathrm{E}-1$ & IMP \\
\hline 5 & I & $1.5935 \mathrm{E}-2$ & $1.3080 \mathrm{E}-2$ & $1.3590 \mathrm{E}-2$ & $1.7503 \mathrm{E}-1$ & EMP \\
\hline 6 & I & $3.3055 \mathrm{E}-2$ & $1.2647 \mathrm{E}-2$ & $1.2651 \mathrm{E}-2$ & $9.9672 \mathrm{E}-2$ & EMP \\
\hline 7 & M & $2.9137 \mathrm{E}-2$ & $4.8776 \mathrm{E}-2$ & $5.0746 \mathrm{E}-2$ & $3.2024 \mathrm{E}-1$ & IMP \\
\hline 8 & M & $4.9367 \mathrm{E}-3$ & $4.5390 \mathrm{E}-3$ & $2.3083 \mathrm{E}-2$ & $3.4027 \mathrm{E}-1$ & IMP \\
\hline 9 & M & $6.1089 \mathrm{E}-3$ & $5.7462 \mathrm{E}-3$ & $1.4978 \mathrm{E}-2$ & $2.0064 \mathrm{E}-1$ & EMP \\
\hline 10 & I & $2.0731 \mathrm{E}-2$ & $2.4169 \mathrm{E}-2$ & $2.4272 \mathrm{E}-2$ & $8.2826 \mathrm{E}-2$ & IMP \\
\hline 11 & I & $1.7674 \mathrm{E}-2$ & $9.4062 \mathrm{E}-3$ & $1.3061 \mathrm{E}-2$ & $2.6923 \mathrm{E}-1$ & EMP \\
\hline 12 & I & $5.0487 \mathrm{E}-2$ & $2.8344 \mathrm{E}-2$ & $3.3369 \mathrm{E}-2$ & $3.3084 \mathrm{E}-1$ & EMP \\
\hline 13 & I & $3.6570 \mathrm{E}-2$ & $3.2976 \mathrm{E}-2$ & $3.8464 \mathrm{E}-2$ & $3.5789 \mathrm{E}-1$ & EMP \\
\hline 14 & I & $1.2273 \mathrm{E}-2$ & $1.1169 \mathrm{E}-2$ & $2.2151 \mathrm{E}-2$ & $3.0842 \mathrm{E}-1$ & EMP \\
\hline 15 & I & $2.8821 \mathrm{E}-2$ & $2.0331 \mathrm{E}-2$ & $2.2494 \mathrm{E}-2$ & $2.7796 \mathrm{E}-1$ & EMP \\
\hline 16 & I & $1.5259 \mathrm{E}-2$ & $2.9673 \mathrm{E}-2$ & $5.5935 \mathrm{E}-2$ & $4.6469 \mathrm{E}-1$ & IMP \\
\hline 17 & I & $5.7718 \mathrm{E}-2$ & $7.3645 \mathrm{E}-2$ & $7.3474 \mathrm{E}-2$ & $1.4339 \mathrm{E}-1$ & IMP \\
\hline ADP & & $1.4983 \mathrm{E}-3$ & $1.1783 \mathrm{E}-3$ & $1.3103 \mathrm{E}-2$ & $2.0209 \mathrm{E}-1$ & IMP \\
\hline
\end{tabular}

Table 6: The minimized sum of square residuals which provides the best fit to 17 sample haloes listed in Tab. 1 together with the averaged density profile (ADP), with regard to the following alternatives: numerical RFSM5 methods involving GPL density profiles (GPN); analytical RFSM5 methods involving GPL density profiles (GPA); analytical RFSM4 methods involving DPL density profiles (DPA); analytical RFSM2 methods involving SPL density profiles i.e. standard least squares method (SPA). Other captions as in Tabs. 2 and 3. 


\begin{tabular}{cccccccc}
\hline \hline case & type & $\alpha$ & \multicolumn{1}{c}{$\chi$} & $\beta$ & \multicolumn{1}{c}{$\gamma$} & class & method \\
\hline 1 & $\mathrm{I}$ & 0.76000 & 21.296 & 17.533 & 1.3480 & IMP & GPA \\
2 & $\mathrm{M}$ & $+\infty$ & 0 & 2.1545 & 1.4338 & BMP & DPA \\
3 & $\mathrm{M}$ & $+\infty$ & 0 & 3.4200 & 1.8638 & IMP & DPA \\
4 & $\mathrm{I}$ & $+\infty$ & 0 & 3.0400 & 1.7367 & IMP & DPA \\
5 & $\mathrm{I}$ & 5.4644 & 0.12476 & 2.3843 & 1.7026 & EMP & GPA \\
6 & $\mathrm{I}$ & 19.787 & 0.027126 & 2.0238 & 1.4870 & EMP & GPA \\
7 & $\mathrm{M}$ & 0.60451 & 4.1915 & 3.5715 & 1.0377 & IMP & GPN \\
8 & $\mathrm{M}$ & 0.42000 & 12.214 & 5.7327 & 0.60284 & IMP & GPA \\
9 & $\mathrm{M}$ & 0.77830 & 2.1159 & 2.7751 & 1.1283 & EMP & GPA \\
10 & $\mathrm{I}$ & 0.43019 & 3.7611 & 2.7372 & 1.1192 & IMP & GPN \\
11 & $\mathrm{I}$ & 3.3124 & 0.28130 & 2.5344 & 1.6026 & EMP & GPA \\
12 & $\mathrm{I}$ & 2.9758 & 0.35736 & 2.0978 & 1.0344 & EMP & GPA \\
13 & $\mathrm{I}$ & 2.1590 & 0.53250 & 2.5151 & 1.3655 & EMP & GPA \\
14 & $\mathrm{I}$ & 0.57290 & 6.0145 & 4.3163 & 0.87060 & EMP & GPA \\
15 & $\mathrm{I}$ & 3.8132 & 0.22865 & 2.2556 & 1.3837 & EMP & GPA \\
16 & $\mathrm{I}$ & 0.74414 & 3.9381 & 3.8674 & 0.93684 & IMP & GPN \\
17 & $\mathrm{I}$ & 0.44450 & 4.0781 & 2.8351 & 1.0224 & IMP & GPN \\
ADP & & 0.29000 & 14.846 & 4.4590 & 1.5360 & IMP & GPA \\
\hline \hline
\end{tabular}

Table 7: Exponents of GPL or DPL density profiles which minimize the sum of square residuals, using both analytical (current paper) and numerical (CMV) RFSM5 or RFSM4 methods, with regard to 17 sample haloes listed in Tab.1, together with the averaged density profile (ADP). Method captions: GPA - analytical RFSM5; GPN - numerical RFSM5; DPA - analytical RFSM4 where GPL density profiles are reduced to their DPL counterparts i.e. $\alpha \rightarrow+\infty$ and $\chi \rightarrow 0$. Other captions as in Tab. 2 . 


\begin{tabular}{ccccccc}
\hline \hline$\xi_{\text {vir }}$ & $r_{0}$ & $\rho_{0}$ & $\sum\left|R_{i}\right|$ & $\sum R_{i}^{2}$ & class & method \\
\hline $8.5258 \mathrm{E}-2$ & $3.2724 \mathrm{E}+4$ & $1.4808 \mathrm{E}-7$ & $2.7556 \mathrm{E}-1$ & $5.3397 \mathrm{E}-3$ & IMP & GPA \\
$9.6963 \mathrm{E}+0$ & $2.9334 \mathrm{E}+2$ & $4.8193 \mathrm{E}-5$ & $6.4395 \mathrm{E}-1$ & $3.5625 \mathrm{E}-2$ & BMP & DPA \\
$1.9336 \mathrm{E}+0$ & $1.4710 \mathrm{E}+3$ & $1.9761 \mathrm{E}-6$ & $9.1288 \mathrm{E}-1$ & $5.1722 \mathrm{E}-2$ & IMP & DPA \\
$1.9743 \mathrm{E}+0$ & $1.4407 \mathrm{E}+3$ & $2.0941 \mathrm{E}-6$ & $7.0852 \mathrm{E}-1$ & $4.3206 \mathrm{E}-2$ & IMP & DPA \\
$7.0536 \mathrm{E}+0$ & $4.0324 \mathrm{E}+2$ & $3.0094 \mathrm{E}-5$ & $3.9790 \mathrm{E}-1$ & $1.3080 \mathrm{E}-2$ & EMP & GPA \\
$2.0441 \mathrm{E}+1$ & $1.2999 \mathrm{E}+2$ & $1.7204 \mathrm{E}-4$ & $3.7559 \mathrm{E}-1$ & $1.2647 \mathrm{E}-2$ & EMP & GPA \\
$4.2267 \mathrm{E}+0$ & $7.4256 \mathrm{E}+2$ & $1.7070 \mathrm{E}-4$ & $6.1250 \mathrm{E}-1$ & $2.9137 \mathrm{E}-2$ & IMP & GPN \\
$5.4741 \mathrm{E}-1$ & $6.4459 \mathrm{E}+3$ & $1.9954 \mathrm{E}-4$ & $2.4863 \mathrm{E}-1$ & $4.5390 \mathrm{E}-3$ & IMP & GPA \\
$9.4211 \mathrm{E}+0$ & $2.7234 \mathrm{E}+2$ & $2.0100 \mathrm{E}-4$ & $2.6901 \mathrm{E}-1$ & $5.7462 \mathrm{E}-3$ & EMP & GPA \\
$6.6374 \mathrm{E}+0$ & $3.6395 \mathrm{E}+2$ & $2.5132 \mathrm{E}-4$ & $5.0765 \mathrm{E}-1$ & $2.0731 \mathrm{E}-2$ & IMP & GPN \\
$6.9351 \mathrm{E}+0$ & $2.8983 \mathrm{E}+2$ & $3.4633 \mathrm{E}-5$ & $3.3590 \mathrm{E}-1$ & $9.4062 \mathrm{E}-3$ & EMP & GPA \\
$1.6379 \mathrm{E}+1$ & $1.6432 \mathrm{E}+2$ & $1.2941 \mathrm{E}-4$ & $4.8093 \mathrm{E}-1$ & $2.8344 \mathrm{E}-2$ & EMP & GPA \\
$8.0514 \mathrm{E}+0$ & $2.4964 \mathrm{E}+2$ & $5.2008 \mathrm{E}-5$ & $6.1515 \mathrm{E}-1$ & $3.2976 \mathrm{E}-2$ & EMP & GPA \\
$1.3437 \mathrm{E}+0$ & $2.0338 \mathrm{E}+3$ & $3.9969 \mathrm{E}-5$ & $3.8299 \mathrm{E}-1$ & $1.1169 \mathrm{E}-2$ & EMP & GPA \\
$8.9712 \mathrm{E}+0$ & $2.6673 \mathrm{E}+2$ & $4.6219 \mathrm{E}-5$ & $4.9331 \mathrm{E}-1$ & $2.0331 \mathrm{E}-2$ & EMP & GPA \\
$3.7154 \mathrm{E}+0$ & $5.8599 \mathrm{E}+2$ & $1.1226 \mathrm{E}-4$ & $4.4819 \mathrm{E}-1$ & $1.5259 \mathrm{E}-2$ & IMP & GPN \\
$4.6774 \mathrm{E}+0$ & $4.9723 \mathrm{E}+2$ & $1.5144 \mathrm{E}-4$ & $7.1010 \mathrm{E}-1$ & $5.7718 \mathrm{E}-2$ & IMP & GPN \\
$2.2073 \mathrm{E}+0$ & $1.2077 \mathrm{E}+3$ & $5.9044 \mathrm{E}-6$ & $1.1027 \mathrm{E}-1$ & $1.1783 \mathrm{E}-3$ & IMP & GPA \\
\hline \hline
\end{tabular}

Table 8: Scale parameters of GPL or DPL density profiles which minimize the sum of square residuals, using both analytical (current paper) and numerical (CMV) RSFM5 or RSFM4 methods, with regard to 17 sample haloes listed in Tab. 1, together with the averaged density profile (ADP). Method captions: GPA - analytical RFSM5; GPN - numerical RFSM5; DPA - analytical RFSM4 where GPL density profiles are reduced to their DPL counterparts i.e. $\alpha \rightarrow+\infty$ and $\chi \rightarrow 0$. Other captions as in Tab. 2. Cases and types have been omitted to save space. 


\begin{tabular}{lrrcccc}
\hline \hline \multicolumn{1}{c}{$\eta$} & \multicolumn{1}{c}{$\left(\eta_{A D P}\right)_{a}$} & \multicolumn{1}{c}{$\left(\eta_{A D P}\right)_{n}$} & $\bar{\eta}$ & $\sigma_{s q}$ & $\sigma_{s \bar{\eta}}$ & $\sigma_{s \bar{\mu}}$ \\
\hline$\alpha$ & $2.9000 \mathrm{E}-1$ & $5.6832 \mathrm{E}-1$ & $3.0190 \mathrm{E}+0$ & $5.8241 \mathrm{E}+0$ & $1.4125 \mathrm{E}+0$ & $2.4225 \mathrm{E}-1$ \\
$\beta$ & $4.4590 \mathrm{E}+0$ & $3.3143 \mathrm{E}+0$ & $3.8702 \mathrm{E}+0$ & $3.6470 \mathrm{E}+0$ & $8.8452 \mathrm{E}-1$ & $1.5169 \mathrm{E}-1$ \\
$\gamma$ & $1.5360 \mathrm{E}+0$ & $1.0000 \mathrm{E}+0$ & $1.3032 \mathrm{E}+0$ & $3.4209 \mathrm{E}-1$ & $8.2969 \mathrm{E}-2$ & $1.4229 \mathrm{E}-2$ \\
$\xi_{\text {vir }}$ & $2.2073 \mathrm{E}+0$ & $3.6308 \mathrm{E}+0$ & $6.5935 \mathrm{E}+0$ & $5.4791 \mathrm{E}+0$ & $1.3289 \mathrm{E}+0$ & $2.2790 \mathrm{E}-1$ \\
$\nu_{\text {mas }}$ & $2.1748 \mathrm{E}-4$ & $5.5823 \mathrm{E}-1$ & $1.4501 \mathrm{E}+1$ & $1.8805 \mathrm{E}+1$ & $4.5608 \mathrm{E}+0$ & $7.8217 \mathrm{E}-1$ \\
$l_{\xi}$ & $3.4386 \mathrm{E}-1$ & $5.6000 \mathrm{E}-1$ & $5.9416 \mathrm{E}-1$ & $5.8669 \mathrm{E}-1$ & $1.4229 \mathrm{E}-1$ & $2.4403 \mathrm{E}-2$ \\
$l_{\nu}$ & $-3.6626 \mathrm{E}+0$ & $-2.5319 \mathrm{E}-1$ & $3.6087 \mathrm{E}-1$ & $1.3833 \mathrm{E}+0$ & $3.3549 \mathrm{E}-1$ & $5.7536 \mathrm{E}-2$ \\
$y_{C}$ & $7.1609 \mathrm{E}+0$ & $4.4000 \mathrm{E}+0$ & $3.9624 \mathrm{E}+0$ & $8.7244 \mathrm{E}-1$ & $2.1160 \mathrm{E}-1$ & $3.6289 \mathrm{E}-2$ \\
$b$ & $1.8564 \mathrm{E}+0$ & $1.8500 \mathrm{E}+0$ & $1.8410 \mathrm{E}+0$ & $1.0135 \mathrm{E}-1$ & $2.4582 \mathrm{E}-2$ & $4.2157 \mathrm{E}-3$ \\
$b_{\beta}$ & $5.6277 \mathrm{E}+0$ & $2.5440 \mathrm{E}+0$ & $3.4029 \mathrm{E}+0$ & $4.4866 \mathrm{E}+0$ & $1.0882 \mathrm{E}+0$ & $1.8662 \mathrm{E}-1$ \\
$b_{\gamma}$ & $7.1081 \mathrm{E}+0$ & $3.8400 \mathrm{E}+0$ & $3.1250 \mathrm{E}+0$ & $7.6132 \mathrm{E}-1$ & $1.8465 \mathrm{E}-1$ & $3.1667 \mathrm{E}-2$ \\
\hline \hline
\end{tabular}

Table 9: Comparison between parameters, $\eta_{A D P}$, related to the best fitting density profile to the averaged SDH density profile (ADP), with regard to analytical and numerical RFSM5 or RFSM4 methods, respectively, and their counterparts, $\bar{\eta}$, averaged over the best fitting density profiles to the whole halo sample, listed in Tabs. 7 and 8. Also listed are the related standard deviations, $\sigma_{s} \eta$, the standard deviations from the mean, $\sigma_{s} \bar{\eta}$, and the standard deviations from the standard deviation from the mean, $\sigma_{s} \bar{\mu}$. With regard to the exponent, $\alpha$, three DPL density profiles have been excluded as $\alpha \rightarrow+\infty$ in that limiting situation. To save space, the notations, $l_{\xi}=\log \xi_{v i r}$ and $l_{\nu}=\log \nu_{\text {mas }}$, have been used. It is worth remembering that $\log \xi_{v i r}=-x_{C}$, according to Eq. (25). 
ent parameters, from less than $\mp \sigma_{s} \bar{\eta}$ to more than $\mp 3 \sigma_{s} \bar{\eta}$. The above result is expected from the theory of the errors.

(xi) The exponents of best fitting, GPL density profiles related to analytical RSFM5 methods, are distant from their NFW counterparts, $(\alpha, \beta, \gamma)=(1,3,1)$, conform to $[\operatorname{Nint}(\alpha), \operatorname{Nint}(\beta), \operatorname{Nint}(\gamma)]=(0,4,2)$, where Nint denotes the nearest integer. The difference increases from about one half for $\gamma$ to about one and half for $\beta$. On the other hand, the contrary holds with regard to GPL density profiles related to numerical RFSM5 methods, namely $[\operatorname{Nint}(\alpha), \operatorname{Nint}(\beta), \operatorname{Nint}(\gamma)]=(1,3,1)$, where the difference increases from about one hundredth for $\gamma$ to about one half for $\alpha(\mathrm{CMV})$.

(xii) The rsm error of the logarithm of the scaled radius, $\xi_{v i r}$, is $\sigma_{s} \log \xi_{v i r} \approx$ 0.60 , to be compared with $\sigma_{s} \log \xi_{v i r}=0.18$ deduced from richer samples where $(\alpha, \beta, \gamma)=(1,3,1) ; M_{v i r}=(0.5-1.0) \times 10^{n} h^{-1} \mathrm{~m}_{\odot} ; 11 \leq n \leq 14$; and $n$ is an integer (Bullock et al. 2001).

\subsection{Discussion}

The application of an analytical RSFM5 method makes a necessary condition for the occurrence of an extremal minimum, Eqs. (36), which is satisfied in 8 sample haloes on a total of 17 , listed in Tab. 1 . The related points in the 5 -dimension parameter hyperspace, $\mathrm{P}^{*} \equiv\left(x_{C}^{*}, y_{C}^{*}, \alpha^{*}, \beta^{*}, \gamma^{*}\right)$, may safely be thought of as absolute extremal minima, by comparison with fiducial minima calculated using a numerical RSFM5 method (CMV). On the other hand, the existence of other extremal minimum points cannot be excluded in absence of a rigorous mathematical proof. In addition, the lack of extremal minima in the remaining 9 sample haloes plus ADP, could be an artifact related to the algorithm used for determining the intersection of the three surfaces, $\gamma=\gamma\left(x_{C}, \alpha\right)$, expressed by Eqs. (36). An improved algorithm would suceed in localizing extremal minimum points in a larger number of sample haloes.

The occurrence of an extremal minimum, or the algorithm power in finding it, seems to be correlated with halo morphology. The relative frequency of the above mentioned event is $7 / 12 \approx 0.583$ for safely relaxed haloes, and $1 / 5=0.2$ for safely undergoing a major merger haloes.

The presence of a (safely absolute) extremal point of minimum, makes a firm criterion for deciding which, among the family of GPL density profiles, 
best fits an assigned SDH density profile. In absence of extremal minima, numerical and analytical RFSM5 or RFSM4 methods, the last using DPL instead of GPL density profiles, seem to be competitive. With regard to sample haloes where no extremal minimum point has been found, the sum of square residuals is minimized using a numerical RFSM5 method in 3 cases, an analytical RFSM5 method in 2 cases, and an analytical RFSM4 method in 3 cases, as can be seen in Tab. 6 .

The related parameters, including cases where an extremal point of minimum occurs, together with ADP, are shown in Tabs.7 and 8. The exponents, $\alpha, \beta, \gamma$, range within $0.42<\alpha<+\infty$ ( $\alpha=0.29$ for ADP); $0<\chi<13(\chi=14.846$ for ADP $) ; 2.0<\beta<17.6 ; 0.6<\gamma<1.9$, respectively. The scaled radius, $\xi_{\text {vir }}$, the scaling radius, $r_{0}$, and the scaling density, $\rho_{0}$, range within $0.08<\xi_{v i r}<20.5 ; 129<r_{0} / \mathrm{kpc}<32724$; $0.001<10^{4} \rho_{0} /\left(10^{10} \mathrm{~m}_{\odot} / \mathrm{kpc}^{3}\right)<2.6$; respectively. The range in inner slope, $\gamma$, is consistent with both core-shaped $(\gamma<1)$ and cuspy $(\gamma \geq 1)$ density profiles ${ }^{3}$. The range in outer slope, $\beta$, is consistent with both infinite $(\beta \leq 3)$ and finite $(\beta>3)$ total (with no truncation) mass.

With regard to the exponent, $\gamma$, it is worth mentioning that, under reasonable boundary conditions such as force-free halo centre and vanishing density at infinite distance, the inequality $\gamma<1$ holds (Mücket \& Hoeft 2003). On the other hand, simple analytic considerations suggest that $\gamma \leq 2$ (Williams et al. 2004). Finally, the validity of Jeans equation implies $1 \leq \gamma \leq 3$ (Hansen 2004), where shallower slopes can arise, provided the effects of baryonic matter are considered (El-Zant et al. 2004; Hansen 2004). The current result, $0.6 \leq \gamma \leq 1.9$, is consistent, or marginally consistent, with the above mentioned constraints.

On the other hand, values of asymptotic inner slopes of fitting density profiles determined in the current paper, are consistent with their counterparts deduced from recent high-resolution simulations using a three-parameter fit involving scaling radius, scaling density, and asymptotic inner slope (6 sample objects, Diemand et al. 2004) or a two-parameter fit involving scaling radius and asymptotic inner slope (16 sample objects, Reed et al. 2004). The related parameters are listed in Tab. 10, which shows agreement between different approaches, within the fiducial range, $\bar{\gamma} \mp 3 \sigma_{s} \bar{\gamma}$. A marginal discrepancy between results from CMV and Reed et al. (2004) can be eliminated, by

\footnotetext{
${ }^{3}$ Strictly speaking, a central cusp occurs for $\gamma>0$.
} 


\begin{tabular}{lrcclccccc}
\hline \hline $\mathrm{c}$ & \multicolumn{1}{c}{$n$} & $\bar{\gamma}$ & \multicolumn{1}{c}{$\sigma_{s \gamma}$} & \multicolumn{1}{c}{$\sigma_{s} \bar{\gamma}$} & \multicolumn{1}{c}{$\Delta^{-} \gamma$} & $\Delta^{+} \gamma$ & $\Delta^{\mp} \gamma$ & $\bar{\gamma}^{-}$ & $\bar{\gamma}^{+}$ \\
\hline $\mathrm{T}$ & 17 & 1.3032 & 0.34209 & 0.082969 & 0.70036 & 0.43350 & 0.56693 & 1.0543 & 1.5521 \\
$\mathrm{E}$ & 8 & 1.3218 & 0.28798 & 0.10181 & 0.45124 & 0.38076 & 0.41600 & 1.0164 & 1.6273 \\
$\mathrm{C}$ & 17 & 1.0018 & 0.13893 & 0.033696 & 0.29868 & 0.19820 & 0.24844 & 0.90071 & 1.1029 \\
$\mathrm{D}$ & 6 & 1.1617 & 0.13732 & 0.056060 & 0.24167 & 0.25833 & 0.25000 & 0.99352 & 1.3299 \\
$\mathrm{R}$ & 16 & 1.2875 & 0.23910 & 0.059774 & 0.28750 & 0.41250 & 0.35000 & 1.1082 & 1.4668 \\
$\mathrm{R}$ & 13 & 1.2692 & 0.24285 & 0.067353 & 0.26923 & 0.43077 & 0.35000 & 1.0671 & 1.4713 \\
\hline \hline
\end{tabular}

Table 10: Comparison between statistical parameters related to the asymptotic inner slope, $\gamma$, deduced from different samples using different fits. Column captions: 1 - case: $\mathrm{T}$ - current paper, all sample haloes; $\mathrm{E}$ - current paper, only sample haloes where an extremum point of minimum occurs; C Caimmi et al. (2004); D - Diemand et al. (2004); R - Reed et al. (2004); 2 $n$ : total number of sample objects; $3-\bar{\gamma}$ : arithmetic mean; $4-\sigma_{s}$ : standard

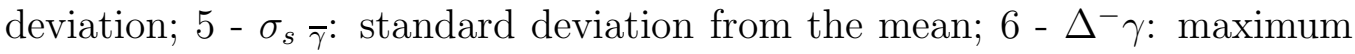
negative deviation from the mean; $7-\Delta^{+} \gamma$ : maximum positive deviation from the mean; $8-\Delta^{\mp} \gamma$ : mean maximum deviation from the mean; 9 -

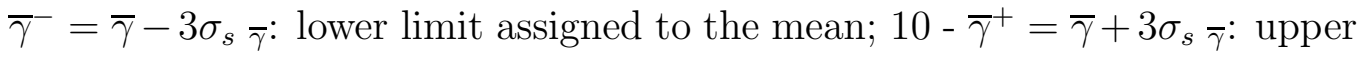
limit assigned to the mean. Sample haloes represent clusters or groups, with the exception of three objects belonging to the richer $\mathrm{R}$ sample (excluded in the poorer R sample), which represent SDHs embedding the Milky Way and two dwarf galaxies, respectively. 
removing from the latter sample three objects, which represent SDHs embedding the Milky Way and two dwarf galaxies, respectively, instead of clusters or groups. On the other hand, current results make an improvement with respect to CMV. Mean values and related scatters, $\bar{\gamma} \mp 3 \sigma_{s} \bar{\gamma}$, corresponding to each case of Tab. 10, are represented in Fig. 1.

No evident correlation is found between SDH dynamical state (relaxed or merging) and asymptotic inner slope of the logarithmic density profile, $-\gamma$, or (for SDH comparable virial masses) scaled radius, $\xi_{v i r}$, contrary to previous results (Ascasibar et al. 2004) related to a sample of 19 high-resolution SDHs on the scale of both clusters of galaxies (13 objects) and galaxies (6 objects), with regard to NFW density profiles. An investigation on richer samples could provide more information to this respect.

The large range in parameters shown in Tabs. 7 and 8, provides further evidence to the occurrence of a certain degree of degeneracy in fitting GPL to SDH density profiles (Klypin et al., 2001; CMV). For instance, with regard to case 14 listed in Tabs. 2 and 3, using analytical and numerical RFSM5 methods, the sum of square residuals changes by less than $10 \%$, while the remaining parameters change by more than $40 \%$.

As different methods are involved, it seems difficult that the occurrence of degeneracy is intrinsic to the procedure of minimization. A remaining possibility could be the lack of resolution in the central regions, which makes the lower limit of the domain, defined by Eq. (14). A more extended (on the left) range, owing to the next generation of high-resolution simulations, could reduce or eliminate the degeneracy. The fit has necessarily to be restricted to the virialized region of the halo, which leaves the upper limit of the domain unchanged.

The occurrence of degeracy could also bias the procedure used for minimizing the sum of any function (in particular, the square) of residuals. For instance, the application of a numerical RFSM5 method to the halo sample listed in Tab. 1 yields an inner slope, $\gamma \approx 1$ (CMV), different from a value $\gamma \approx 1.5$ currently found for dark matter haloes on the scale of cluster of galaxies (e.g., Fukushige \& Makino 2003; Hiotelis 2003), but closer to $\gamma \approx 1.2-1.3$ from more recent attempts (Diemand et al. 2004; Reed et al. 2004). On the other hand, the application of an analytical RFSM5 method to the above mentioned halo sample yields an inner slope, $\gamma \approx 1.5$ (current paper).

The scaled radius, $\xi_{v i r}=r_{v i r} / r_{0}$, is defined in the current paper as related to the point where the maximum change in logarithmic slope occurs 


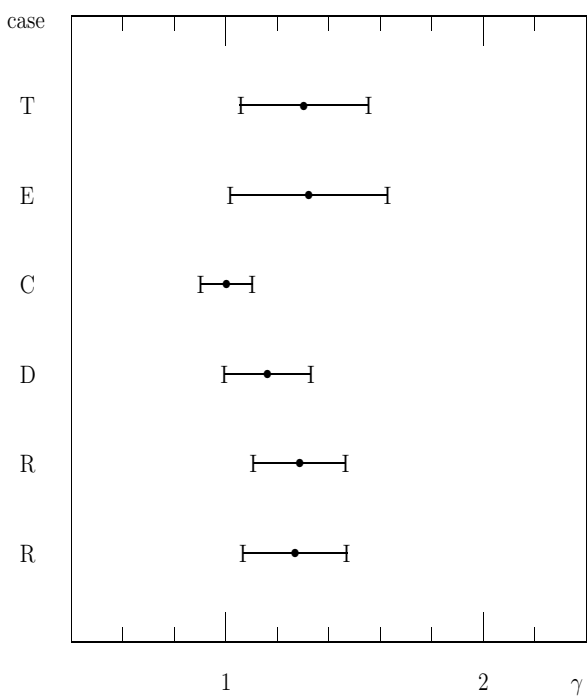

Figure 1: Mean values and related scatters, $\bar{\gamma} \mp 3 \sigma_{s} \bar{\gamma}$, corresponding to cases listed in Tab. 10, positioned and labeled as therein. 
in the corresponding GPL density profile (CMV). On the other hand, the concentration, $c=r_{v i r} / r_{-2}$, is usually defined as related to the point where the logarithmic slope equals -2 (e.g., Klypin et al. 2001; Bullock et al. 2001; Hiotelis 2003). The former definition seems to be more general, as it provides the scaled radius meaningful also in early times, where the slope of a GPL density profile may be smaller (in absolute value) than 2, while the contrary holds for the concentration (Hiotelis 2003).

The GPL density profile which best fits to the averaged SDH density profile, with regard to analytical RFSM5 methods, is characterized by exponents, $(\alpha, \beta, \gamma)$, satisfying $[\operatorname{Nint}(\alpha), \operatorname{Nint}(\beta), \operatorname{Nint}(\gamma)]=(0,4,2)$, which is different from NFW density profiles currently used in literature, $(\alpha, \beta, \gamma)=(1,3,1)$. The comparison with values averaged over the whole halo sample, discloses that fluctuations of the exponents around their NFW counterparts may safely be excluded. Accordingly, NFW density profiles cannot be conceived as intrinsic to dark matter haloes of the current sample.

On the other hand, following e.g., Bullock et al. (2001), NFW density profiles (or alternative functional forms) may be considered as a convenient way to parametrize SDH density profiles, without implying that it necessarily provides the best possible fit. This is why the scaled radius, $\xi_{\text {vir }}$, and the scaled mass, $\nu_{\text {mas }}$, can be interpreted as general structure parameters, not necessarily restricted to a specific density profile. In particular, any spread in $\xi_{v i r}$ and $\nu_{\text {mas }}$ can be attributed to a real (larger) scatter in a "physical" scaling radius, defined by e.g., the radius where the change in slope of the logarithmic density profile attains its maximum value (CMV), rather than to inaccuracies in an assumed, "universal" density profile. For further details see e.g., Bullock et al. (2001).

Additional support to the above considerations is provided by the value calculated for the standard deviation of the decimal logarithm of the scaled radius, $\sigma_{s \log \xi_{v i r}} \approx 0.59$, which is 3.3-3.4 times larger than $\sigma_{s \log \xi_{\text {vir }}} \approx 0.18$ deduced from a statistical sample of about five thousands simulated haloes, within mass bins equal to $(0.5-1.0) \times 10^{n} h^{-1} \mathrm{~m}_{\odot}$, where $11 \leq n \leq 14$ and $n$ is an integer, with regard to NFW density profiles (Bullock et al. 2001). A comparable scatter, $\sigma_{s \log \xi_{v i r}} \approx 0.17$, has been obtained using numerical RSFM5 methods (CMV). The discrepancy with the results of the current paper, is probably owing to a bias in the selection of the subdomain where numerical RFSM5 methods work.

To get further insight, the histogram of the decimal logarithm of the scaled 
radius, $\log \xi_{\text {vir }}$, is represented in Fig. 2, with regard to numerical (CMV) and best fitting (current paper, Tab. 8) RSFM5 methods. The former case (top panel) shows a clear cut at $\log \xi_{v i r}=0.55$, which could be a biasing effect, as mentioned above, but the dispersion is low. The latter case (bottom panel) shows no sign of cut, but the dispersion is large. It is reduced only slightly by restricting the calculations to a homogeneous subsample, where only EMPs are considered, and even if the maximum deviation from the arithmetic mean is excluded therein. If a large dispersion still maintains for richer samples $(N \gg 17)$, in dealing with analytical RSFM5 methods, a low dispersion found in $N$-body simulations (e.g., Bullock et al. 2001) seems to be an artefact, related to the assumption of NFW (or any equivalent choice) density profile.

On the other hand, a large spread in parameters of GPL density profiles where an extremum point of minimum occurs, may be considered as fiducial at most. This is in absence of a rigorous proof on the existence of an absolute point of minimum. If, in fact, absolute minima do not coincide with the extremum points of minimum which are found in the current attempt, then the spread in parameters could be reduced.

The results of the current paper confirm a certain degree of degeneracy in fittig GPL to SDH density profiles, as pointed out by Klypin et al. (2001). For instance, cases 4 and 14 of Tabs. 2 and 3 exhibit - on the related upper and lower lines - values of the sum of square residuals and absolute value residuals, which are close each to the other. On the contrary, the difference substantially grows for the remaining parameters. As degeneracy occurs using analytical and numerical RFSM5 methods separately, it cannot safely be considered as an artifact of the corresponding algorithm. It might be either a real feature of the problem of minimization, or a consequence of an incomplete domain, towards central regions, of SDH density profiles, owing to numerical artifacts (mainly two-body relaxation). The latter possibility could be tested by the next generation of high-resolution simulations.

The current attempt is limited to GPL density profiles, defined by Eq. (1), but different alternatives have been exploited in the literature, such as the family of density profiles:

$$
\rho\left(\frac{r}{r_{0}}\right)=\rho_{0}\left[1+\left(\frac{r}{r_{0}}\right)^{\alpha}\right]^{-\chi}\left[1+\frac{r}{r_{0}}\right]^{-\gamma} ; \quad \chi=\frac{\beta-\gamma}{\alpha} ;
$$

where the choice $(\alpha, \beta, \gamma)=(2,3,1)$ corresponds to the Burkert (1995) density profile, which resembles the NFW density profile for $r \gtrsim 0.02 r_{v i r}$ (e.g. 

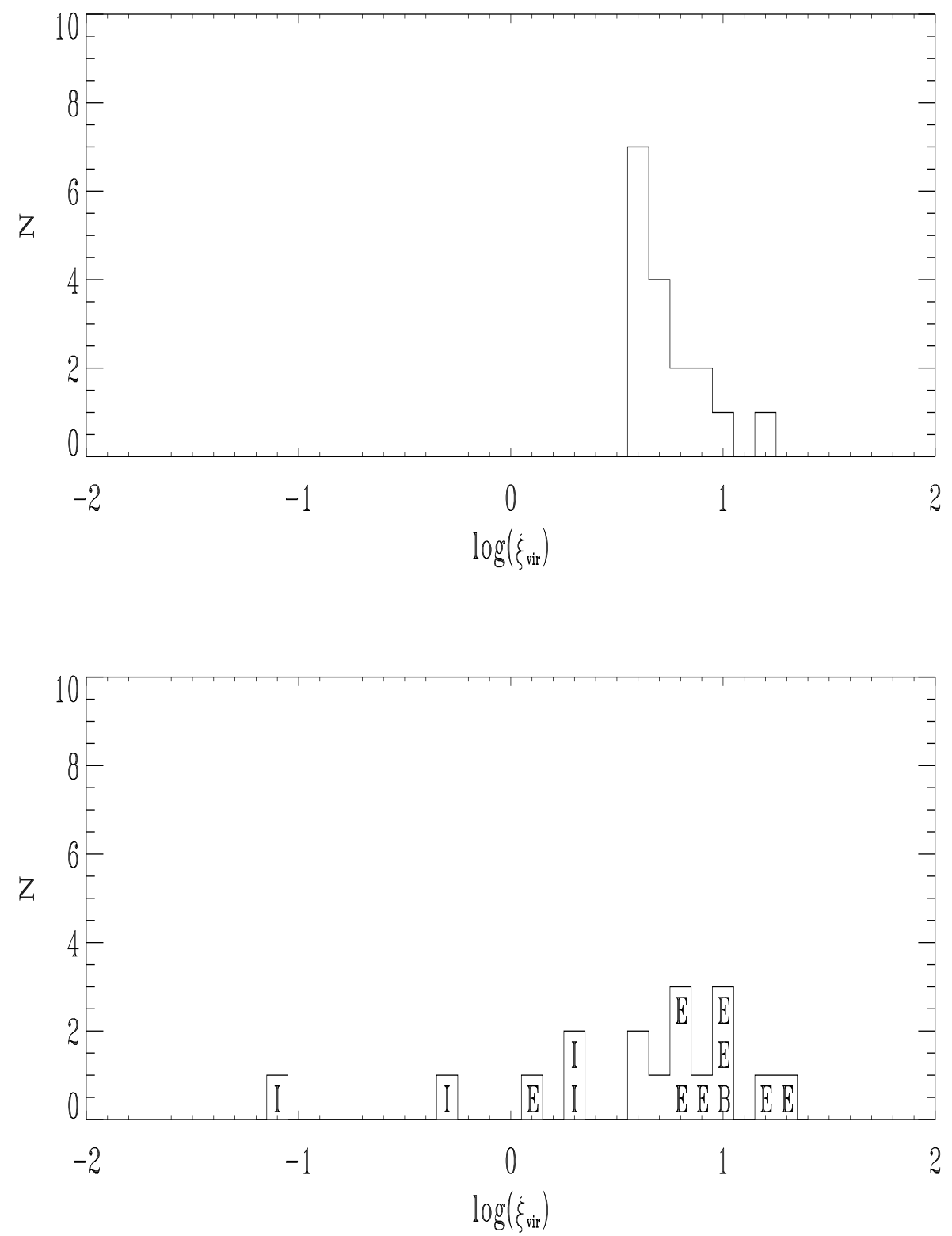

Figure 2: Histogram of the decimal logarithm of the scaled radius, $\log \xi_{\text {vir }}$, with regard to numerical (CMV; top) and best fitting (current paper, Tab. 8; bottom) RSFM5 methods. Label captions: I-IMP; E-EMP; B-BMP; blank numerical (CMV) RSFM5 methods i.e. same cases as in the upper istogram. 
Bullock et al. 2001).

Another possibility is a profile that curves smoothly over to a constant density at very small radii (Navarro et al. 2004):

$$
\rho=\rho_{0} \exp \left\{-\frac{2}{\lambda}\left[\left(\frac{r}{r_{0}}\right)^{\lambda}-1\right]\right\} ;
$$

where the parameter, $\lambda$, prescribes how fast the density profile turns away from a power-law near the centre. The best fit reads (19 sample objects, Navarro et al. 2004): $\bar{\lambda} \mp 3 \sigma_{s} \bar{\lambda}=0.17216 \mp 0.021897$.

On the other hand, the RFSM5 method may be extended to any kind of density profile.

\section{Conclusion}

Analytical and geometrical properties of generalized power-law (GPL) density profiles have been reviewed, and special effort has been devoted to the special cases where GPL density profiles reduce to (i) a double power-law (DPL), and (ii) a single power-law (SPL). Then GPL density profiles have been compared with simulated dark haloes (SDH) density profiles, and nonlinear least-squares fits have been prescribed, involving five parameters (a scaling radius, $r_{0}$, a scaling density, $\rho_{0}$, and three exponents, $\alpha, \beta, \gamma$ ), which specify the fitting profile (RFSM5 method).

More specifically, the validity of a necessary condition for the occurrence of an extremum point, has been related to the existence of an intersection between three surfaces, $\gamma_{k}=f_{k}\left(r_{0}, \alpha\right), k=1,2,3$, in a 3-dimension space, $\left(\mathrm{O} r_{0} \alpha \gamma\right)$. Using the algorithm has made also establish that the extremum point is a fiducial minimum, while the explicit calculation of the Hessian determinant has been avoided to gain in simplicity. In absence of a rigorous proof, the fiducial minimum could be considered as nothing but a fiducial absolute minimum.

An application has been made to a sample of 17 SDHs on the scale of cluster of galaxies, within a flat $\Lambda \mathrm{CDM}$ cosmological model (Rasia et al. 2004). In dealing with the averaged SDH density profile (ADP), a virial radius, $r_{v i r}$, equal to the mean over the whole sample, has been assigned, which has allowed the calculation of the remaining parameters. The following results have been found. (i) A necessary condition for the occurrence of an 
extremum point is satisfied for 8 sample haloes, and is not for the remaining 9 together with ADP. In the former alternative, an extremum point of minimum (EMP) may safely exist. In the latter alternative, the occurrence of an EMP cannot be excluded, but only a local minimum can be determined. (ii) The occurrence of an EMP implies a sum of square residuals which is systematically lower than its counterpart deduced by use of numerical RFSM5 methods. Accordingly, EMPs may safely be thought of as absolute minima. With regard to sample haloes where no EMP is detected, the above result maintains in 4 cases (including ADP), while the contrary holds for the remaining 5 cases. (iii) The best fit (with no EMP detected) is provided by DPL density profiles for 3 sample haloes. In addition, DPL density profiles make a rough, but viable approximation in fitting SDH density profiles. The contrary holds for SPL density profiles.

No evident correlation has been found between SDH dynamical state (relaxed or merging) and asymptotic inner slope of the logarithmic density profile or (for SDH comparable virial masses) scaled radius.

Mean values and standard deviations of some parameters have been calculated and, in particular, the decimal logarithm of the scaled radius, $\xi_{\text {vir }}$, has read $<\log \xi_{v i r}>\approx 0.59$ and $\sigma_{s \log \xi_{v i r}} \approx 0.59$, the standard deviation exceeding by a factor 3.3-3.4 its counterpart evaluated in an earlier attempt using NFW density profiles (Bullock et al. 2001). If a large dispersion still maintains for richer samples, in dealing with analytical RSFM5 methods, a low dispersion found in $N$-body simulations has seemed to be an artefact, due to the assumption of NFW (or any equivalent choice) density profile. It has provided additional support to the idea, that NFW density profiles may be considered as a convenient way to parametrize SDH density profiles, without implying that it necessarily produces the best possible fit (Bullock et al. 2001).

With regard to RFSM5 methods formulated in the current paper, the exponents of both the best fitting GPL density profile to $\operatorname{ADP},(\alpha, \beta, \gamma) \approx$ $(0.3,4.5,1.5)$, and related averages calculated over the whole halo sample, $(\bar{\alpha}, \bar{\beta}, \bar{\gamma}) \approx(3.0,3.9,1.3)$, have been found to be far from their NFW counterparts, $(\alpha, \beta, \gamma)=(1,3,1)$. The last result, together with the large value of the standard deviation, $\sigma_{s} \log \xi_{v i r}$, has been interpreted as due to a certain degree of degeneracy in fitting GPL to SDH density profiles. If it is a real feature of the problem, or it could be reduced by the next generation of high-resolution simulations, still has remained an open question. Values of asymptotic inner 
slope of fitting logarithmic density profiles, have been found to be consistent with results from recent high-resolution simulations (Diemand et al. 2004; Reed et al. 2004).

\section{Acknowledgements}

We are endebted with E. Rasia, G. Tormen, and L. Moscardini, for making the results of their simulations available to us. In addition, we are deeply grateful to all of them for clarifying and fruitful discussions.

\section{References}

[1] Ascasibar, Y., Yepes, G., Gottlöber, S., Müller, V. 2003. MRNAS 352, 1109

[2] Bullock, J. S., Kolatt, T. S., Sigad, Y., et al. 2001, MNRAS 321, 559

[3] Burkert, A. 1995, ApJ 447, L25

[4] Caimmi, R., Marmo, C. 2003, NewA 8, 119

[5] Caimmi, R., Marmo, C., Valentinuzzi, T. 2004, astro-ph/0411455 (CMV)

[6] Cole, S., Lacey, C., 1996, MNRAS 281, 716

[7] Diemand, J., Moore B., Stadel, J. 2004, MNRAS 353, 624

[8] El-Zant, A.A., Hoffman, J., Primack, J., Combes, F., Sloshman, I. 2004, ApJ 607, L75

[9] Fukushige, F., Makino, J. 2001, ApJ 557, 533

[10] Fukushige, F., Makino, J. 2003, ApJ 588, 674

[11] Fukushige, F., Kawai, A., Makino, J. 2004, ApJ 606, 625

[12] Hansen, S.H. 2004, MNRAS 352, L41

[13] Hernquist, L. 1990, ApJ 356, 359 
[14] Hiotelis, N. 2003, MNRAS 344, 149

[15] Huss, A., Jain, B., Steinmetz, M. 1999, ApJ 517, 64

[16] Jing, Y.P., Suto, Y. 2000, ApJ 529, L69

[17] Klypin, A., Kravtsov, A.V., Bullok, J.S., Primack, J.R. 2001, ApJ 554, 903

[18] Moore, B., Governato, F., Quinn, T., Stadel, J., Lake, G. 1998, ApJ 499, L5

[19] Moore, B., Quinn, T., Governato, F., Stadel, J., Lake, G. 1999, MNRAS 310,1147

[20] Mücket, J.P., Hoeft, M. 2003, A\&A 404, 809

[21] Navarro, J.F., Frenk, C.S., White, S.D.M. 1995, MNRAS 275, 720

[22] Navarro, J.F., Frenk, C.S., White, S.D.M. 1996, ApJ 462, 563

[23] Navarro, J.F., Frenk, C.S., White, S.D.M. 1997, ApJ 490, 493

[24] Navarro, J.F., Hagashi, E., Power, C., et al. 2004, MNRAS 349, 1039

[25] Oliva, P.R., Terrasi, F. 1976. Elaborazione Statistica dei Risultati Sperimentali, Liguori Editore, Napoli

[26] Rasia, E., Tormen, G., Moscardini, L. 2004, MNRAS 351, 237

[27] Reed, D., Governato, F., Verde, L., et al. 2004, astro-ph/0312544

[28] Ricotti, M. 2003, MNRAS 344, 1237

[29] Rubiño-Martin, J.A., Rebolo, R., Carreira, P., et al. 2003, MNRAS 341, 1084

[30] Sievers, J.L., Bond, J.R., Cartwright, J.K., et al. 2003, ApJ 591, 599

[31] Spergel, D.N., Verde, L., Peiris, H.V., et al. 2003, ApJSupp 148, 175

[32] Syer, D., White, S. D. M. 1998, MNRAS 293, 337 
[33] Smirnov, V., 1969, Cours de Matematiques Superieures, vol.I, Mir, Moscow

[34] Tasitsiomi, A., Kravstov, A.V., Gottlöeber, S., Klypin, A.A. 2004 ApJ 607,125

[35] Williams, L.L.R., Babul, A., Dalcanton, J.J. 2004, ApJ 604, 18

[36] Zhao, D.H., Mo, H.J., Jing, J.P., Börner, G. 2003, MNRAS 339, 12

\section{A On the occurrence of extremum points of minimum to the sum of square residuals}

A necessary condition for the occurrence of extremum points of minimum to the sum of square residuals, defined by Eq. (34), is expressed by Eqs. (35), or more explicitly as:

$$
\begin{aligned}
& \sum_{i=1}^{n} R_{i} \frac{\partial R_{i}}{\partial x_{C}}=0 ; \\
& \sum_{i=1}^{n} R_{i} \frac{\partial R_{i}}{\partial y_{C}}=0 ; \\
& \sum_{i=1}^{n} R_{i} \frac{\partial R_{i}}{\partial \alpha}=0 ; \\
& \sum_{i=1}^{n} R_{i} \frac{\partial R_{i}}{\partial \beta}=0 ; \\
& \sum_{i=1}^{n} R_{i} \frac{\partial R_{i}}{\partial \gamma}=0 ;
\end{aligned}
$$

where, for sake of simplicity, it is intended that the residuals and their first derivatives are calculated at a fiducial extremum point of minimum, $\mathrm{P} \equiv$ $\left(x_{C}^{*}, y_{C}^{*}, \alpha^{*}, \beta^{*}, \gamma^{*}\right)$.

By use of Eq. (31), the first derivatives of the $i$-th residual read:

$$
\frac{\partial R_{i}}{\partial x_{C}}=-\gamma-(\beta-\gamma) t_{i} ;
$$




$$
\begin{aligned}
& \frac{\partial R_{i}}{\partial y_{C}}=-1 ; \\
& \frac{\partial R_{i}}{\partial \alpha}=-\frac{\beta-\gamma}{\alpha^{2}} s_{i}+\frac{\beta-\gamma}{\alpha}\left(x_{i}-x_{C}\right) t_{i} ; \\
& \frac{\partial R_{i}}{\partial \beta}=\frac{1}{\alpha} s_{i} ; \\
& \frac{\partial R_{i}}{\partial \gamma}=\left(x_{i}-x_{C}\right)-\frac{1}{\alpha} s_{i} ;
\end{aligned}
$$

where both $s_{i}$ and $t_{i}$ depend on $w_{i}=\exp _{10}\left[\alpha\left(x_{i}-x_{C}\right)\right]$, according to Eqs. (38a) and (38b), respectively. In addition, the validity of the relations:

$$
\begin{aligned}
& \frac{\partial R_{i}}{\partial \gamma}+\frac{\partial R_{i}}{\partial \beta}=\left(x_{i}-x_{C}\right) ; \\
& \frac{\partial R_{i}}{\partial \alpha}+\frac{x_{i}-x_{C}}{\alpha} \frac{\partial R_{i}}{\partial x_{C}}+\frac{\beta-\gamma}{\alpha} \frac{\partial R_{i}}{\partial \beta}=-\frac{\gamma}{\alpha}\left(x_{i}-x_{C}\right) ;
\end{aligned}
$$

may easily be checked.

The combination of Eqs. (48b) and (49b) yields:

$$
\sum_{i=1}^{n} R_{i}=0
$$

and the combination of Eqs. (48d), (48e), (50), and (52), yields:

$$
\sum_{i=1}^{n} R_{i} x_{i}=0
$$

on the other hand, multiplying the left and the right-hand side of Eq. (51) by $R_{i}$ and summing over all the residuals, produces:

$$
\begin{aligned}
& \sum_{i=1}^{n} R_{i} \frac{\partial R_{i}}{\partial \alpha}+\sum_{i=1}^{n} \frac{x_{i}-x_{C}}{\alpha} R_{i} \frac{\partial R_{i}}{\partial x_{C}} \\
& +\frac{\beta-\gamma}{\alpha} \sum_{i=1}^{n} R_{i} \frac{\partial R_{i}}{\partial \beta}=-\frac{\gamma}{\alpha} \sum_{i=1}^{n} R_{i}\left(x_{i}-x_{C}\right) ;
\end{aligned}
$$

which, owing to Eqs. (48a), (48c), (48d), (52), and (53), yields:

$$
\sum_{i=1}^{n} x_{i} R_{i} \frac{\partial R_{i}}{\partial x_{C}}=0
$$


similarly, the combination of Eqs. (48a), (49a), (52), and (55), yields:

$$
\sum_{i=1}^{n} R_{i} t_{i}=0
$$

and the combination of Eqs. (48d) and (49d) yields:

$$
\sum_{i=1}^{n} R_{i} s_{i}=0
$$

finally, the combination of Eqs. (48c), (49c), (56), and (57), yields:

$$
\sum_{i=1}^{n} R_{i} x_{i} t_{i}=0
$$

in conclusion, Eqs. (52), (53), (56), (57), and (58), make a system of five independent equations which is equivalent to (48).

The substitution of Eqs. (31) and (38a) into (52) yields:

$$
\left(\bar{y}-y_{C}\right)+\gamma\left(\bar{x}-x_{C}\right)+\frac{\beta-\gamma}{\alpha} \bar{s}=0 ;
$$

where overbars denote mean values, according to Eq. (37b). The comparison between alternative expressions of $y_{C}$, deduced from Eqs. (31) and (59) produces:

$$
R_{i}=\left(y_{i}-\bar{y}\right)+\gamma\left(x_{i}-\bar{x}\right)+\frac{\beta-\gamma}{\alpha}\left(s_{i}-\bar{s}\right) ;
$$

where no dependence on $y_{C}$ occurs.

The substitution of Eq. (60) into (53) yields:

$$
v_{s}(x, y)+\gamma v_{s}(x, x)+\frac{\beta-\gamma}{\alpha} v_{s}(x, s)=0
$$

where the function, $v_{s}$, is defined by Eqs. (37), and can be conceived as a two-dimension (2D) empirical variance. This is why, in the limit of a onedimension (1D) empirical variance, $v_{s}(a, a)=\overline{\left(a^{2}\right)}-(\bar{a})^{2}$, according to the standard notation. It is worth of note that, while $1 \mathrm{D}$ empirical variances are always non negative, the contrary holds for $2 \mathrm{D}$ empirical variances. 
The comparison between alternative expressions of $\chi=(\beta-\gamma) / \alpha$, deduced from Eqs. (60) and (61), produces:

$$
\begin{aligned}
R_{i}= & \left(y_{i}-\bar{y}\right)+\gamma\left(x_{i}-\bar{x}\right) \\
& -\frac{v_{s}(x, y)-\gamma v_{s}(x, x)}{v_{s}(x, s)}\left(s_{i}-\bar{s}\right) ;
\end{aligned}
$$

where no dependence on $\beta$ occurs. Then the combination of Eqs. (56) and (62) yields:

$$
\begin{aligned}
& v_{s}(x, s) v_{s}(y, t)-v_{s}(s, t) v_{s}(x, y)= \\
& \gamma\left[v_{s}(s, t) v_{s}(x, x)-v_{s}(x, s) v_{s}(x, t)\right] ;
\end{aligned}
$$

and the combination of Eqs. (62) and (63) allows an expression of $R_{i}$ which depends only on $x_{C}$ and $\alpha$, via Eqs. (38a) and (38b).

The combination of Eqs. (57) and (62) yields:

$$
\begin{aligned}
& v_{s}(x, s) v_{s}(y, s)-v_{s}(s, s) v_{s}(x, y)= \\
& \gamma\left[v_{s}(s, s) v_{s}(x, x)-v_{s}(x, s) v_{s}(x, s)\right] ;
\end{aligned}
$$

which has the same formal expression as Eq. (63), provided the following correspondences hold: $(y, t) \leftrightarrow(y, s) ;(s, t) \leftrightarrow(s, s) ;(x, t) \leftrightarrow(x, s)$.

The combination of Eqs. (58) and (62) yields:

$$
\begin{aligned}
& v_{s}(x, s) v_{s}(y, x t)-v_{s}(s, x t) v_{s}(x, y)= \\
& \gamma\left[v_{s}(x, x) v_{s}(s, x t)-v_{s}(x, s) v_{s}(x, x t)\right] ;
\end{aligned}
$$

which has the same formal expression as Eqs. (63), and (64), provided the following correspondences hold: $(y, t) \leftrightarrow(y, s) \leftrightarrow(y, x t) ;(s, t) \leftrightarrow(s, s) \leftrightarrow$ $(s, x t) ;(x, t) \leftrightarrow(x, s) \leftrightarrow(x, x t)$.

Then we are left with three alternative expressions of $\gamma$, which may be deduced from Eqs. (63), (64), (65), and are expressed by Eqs. (36a), (36b), (36c), respectively.

A necessary condition for the occurrence of extremum points of minimum to the sum of square residuals, defined by Eq. (34), has been formulated via Eqs. (48). The condition is also sufficient, provided the Hessian determinant 
satisfies Eq. (33). The derivation of both sides of Eq. (35) yields:

$$
\begin{aligned}
& \left(\frac{\partial^{2} F}{\partial u_{j}^{2}}\right)_{\mathrm{P}^{*}}=2 \sum_{i=1}^{n}\left[\left(\frac{\partial R_{i}}{\partial u_{j}}\right)_{\mathrm{P}^{*}}^{2}+R_{i}\left(\frac{\partial^{2} R_{i}}{\partial u_{j}^{2}}\right)_{\mathrm{P}^{*}}\right] ; \\
& \left(\frac{\partial^{2} F}{\partial u_{j} u_{k}}\right)_{\mathrm{P}^{*}}=2 \sum_{i=1}^{n}\left[\left(\frac{\partial R_{i}}{\partial u_{j}}\right)_{\mathrm{P}^{*}}\left(\frac{\partial R_{i}}{\partial u_{k}}\right)_{\mathrm{P}^{*}}+R_{i}\left(\frac{\partial^{2} R_{i}}{\partial u_{j} \partial u_{k}}\right)_{\mathrm{P}^{*}}\right] ;
\end{aligned}
$$

where $u_{1}=x_{C}, u_{2}=y_{C}, u_{3}=\alpha, u_{4}=\beta, u_{5}=\gamma$.

The second derivatives of the $i$-th residuals may be determined, using Eqs. (38a), (38b), (38c), (39a), (39b), and (49). The result is:

$$
\begin{aligned}
& \frac{\partial^{2} R_{i}}{\partial x_{C}^{2}}=\alpha(\beta-\gamma) \frac{t_{i}}{w_{i}} \ln 10 ; \\
& \frac{\partial^{2} R_{i}}{\partial y_{C}^{2}}=0 ; \\
& \frac{\partial^{2} R_{i}}{\partial \gamma^{2}}=0 ; \\
& \frac{\partial^{2} R_{i}}{\partial \beta^{2}}=0 ; \\
& \frac{\partial^{2} R_{i}}{\partial \alpha^{2}}=\frac{\beta-\gamma}{\alpha} \times \\
& \left.\frac{2}{\alpha^{2}} s_{i}-\frac{2}{\alpha}\left(x_{i}-x_{C}\right) t_{i}+\left(x_{i}-x_{C}\right)^{2} \frac{t_{i}^{2}}{w_{i}} \ln 10\right] ; \\
& \frac{\partial^{2} R_{i}}{\partial y_{C} \partial x_{C}}=0 ; \\
& \frac{\partial^{2} R_{i}}{\partial y_{C} \partial \gamma}=0 ; \\
& \frac{\partial^{2} R_{i}}{\partial y_{C} \partial \beta}=0 ; \\
& \frac{\partial^{2} R_{i}}{\partial y_{C} \partial \alpha}=0 ; \\
& \frac{\partial^{2} R_{i}}{\partial \gamma \partial \alpha}=\frac{1}{\alpha^{2}} s_{i}-\frac{x_{i}-x_{C}}{\alpha} t_{i} ; \\
& \frac{\partial^{2} R_{i}}{\partial \gamma \partial x_{C}}=-\frac{t_{i}}{w_{i}} ;
\end{aligned}
$$




$$
\begin{aligned}
& \frac{\partial^{2} R_{i}}{\partial \beta \partial x_{C}}=-t_{i} ; \\
& \frac{\partial^{2} R_{i}}{\partial \beta \partial \alpha}=-\frac{1}{\alpha^{2}} s_{i}+\frac{x_{i}-x_{C}}{\alpha} t_{i} ; \\
& \frac{\partial^{2} R_{i}}{\partial x_{C} \partial \alpha}=-(\beta-\gamma)\left(x_{i}-x_{C}\right) \frac{t_{i}^{2}}{w_{i}} \ln 10 ;
\end{aligned}
$$

which allows the calculation of the Hessian determinant. The related, analytical expression appears to be extremely complicated, and for this reason it shall not be calculated here.

In principle, the above procedure could be used for minimizing the sum of absolute value residuals, but at a higher price. This is why the first derivatives of the function:

$$
F\left(x_{C}, y_{C}, \alpha, \beta, \gamma\right)=\sum_{i=1}^{n}\left|R_{i}\right|
$$

are not defined at $R_{i}=0,1 \leq i \leq n$, which makes a necessary condition for the existence of an absolute minimum, expressed by Eq. (32), satisfied at any point, $\mathrm{P}^{*}\left(x_{C}^{*}, y_{C}^{*}, \alpha^{*}, \beta^{*}, \gamma^{*}\right)$, where $R_{i}=0$. To avoid this inconvenient, our attention has been restricted to the minimization of the sum of square residuals. 